\title{
HISTOIRE DES IDEES RELIGIEUSES ET PHILOSOPHIQUES
}

Athéisme et agnosticisme. Colloque de Bruxelles, mai 1986, éd. par Jacques MARX. Bruxelles, Éd. de l'Université de Bruxelles, 1987. 16 × 24, 183 p. (« Problèmes d'histoire du christianisme ", 16).

Ce volume rassemble les communications présentées au Colloque international organisé les 15 et 16 mai 1986 par l'Institut des religions et de la laïcité de l'Université libre de Bruxelles.

Après une Introduction par André Jaumotte (p. 7-10), on y trouve d'abord quatre communications d'ordre historique : "L'athéisme jugé par les chrétiens des premiers siècles " par Hervé Savon (p. 11-24), "Le problème religieux dans les voyages imaginaires au seuil des Lumières " par Raymond Trousson (p. 25 43), « L'athéisme en France au XVIIr siècle : progrès et résistances " par Roland Mortier (p. 45-62), "Réflexions sur l'athéisme chez Marx et dans le marxisme " par Guy Haarscher (p. 63-72). Sans être étrangères à l'histoire, les communications suivantes sont d'ordre plus théorique, allant de la sociologie (cf. « Athéisme, socialisme, sociologie " par Georges Goriely, p. 89-97, et " Filtres idéologiques et anthropologiques chez des "croyants" et des "non-croyants" " par JeanPierre Deconchy, p. 99-119) à l'épistémologie ("De la science athée à la technique agnostique " par Gilbert Hottois, p. 133-142), la morale (" Athéisme et morale " par Henri Janne, p. 121-132, "Secular Humanism " par Paul Kurtz, p. 143-150), la métaphysique (" Dieu est mort, l'homme est mort, et que faire?" par Michel Meyer, p. 73-87, « Les apories de l'ontologie » par Jean Ladrière, p. 151-165), et la théologie ("Athéisme et agnosticisme" par Léo Apostel, p. 167-178).

Très ouvert tant par la diversité des positions qui s'y expriment que par les perspectives qu'il trace, comme y insiste en guise de conclusion Robert Joly ( "Ce n'est qu'un au-revoir », p. 179-182), un tel volume est, comme il est naturel, d'une grande inégalité, de ton et de caractère tout au moins. Si les deux premières communications retiennent l'attention par la richesse de précision et d'instruction qu'elles foumissent dans leur brièveté même, si la troisième est une vigoureuse synthèse sur l'athéisme au siècle des Lumières par un des meilleurs spécialistes

Revue de synthèse: IV S. No 2, avril-juin 1988. 
de celui-ci, d'autres paraîtront sans doute plus sommaires ou évasives, plus suggestives parfois que convaincantes. Dans un tel ensemble, l'allure savante d'une communication comme celle de $M$. Deconchy doit-elle vraiment impressionner, ou bien faut-il retenir des considérations d'apparence très technique, par lesquelles elle s'ouvre et se clôt, que la non-croyance n'est pas une croyance, et si c'est le cas, fallait-il un tel appareillage pour en arriver là?

Il est vrai qu'une telle interrogation s'enracine, plus généralement, dans le terrain sur lequel se situe $œ$ débat, marqué qu'il est du sceau de l'antinomie par le nom de l'Institut qui l'organisait, comme par le titre et le sous-titre sous lesquels en sont publiés les Actes, situation qui peut tout naturellement conduire un des auteurs à proposer à "l'athée actuel " de "trouver une attitude religieuse " (p. 177) : explicite ou implicite, c'est bien en effet la présupposition d'une bonne partie des intervenants que d'appréhender en termes religieux les attitudes et courants pris ici en considération, ce qui correspond, il est vrai, à des termes comme ceux d" " athéisme " et d" "agnosticisme ", qui portent en eux la marque des liens que, bon gré mal gré, les affranchis de la religion continuent en un premier temps d'entretenir avec leur ancien joug.

Dans ces limites, et malgré l'ambiguỉté qui caractérise ainsi certains des textes ici rassemblés, on y trouvera un ensemble de données et de considérations qui donnent à réfléchir sur les attitudes irréligieuses, philosophiques et autres, leur histoire, les questions qu'elles peuvent aujourd'hui poser, ou se poser, et celles qui se posent à partir d'elles.

Olivier BLOCH.

Proclus, Sur le Premier Alcibiade de Platon. T. I et II. Texte établi et traduit par Alain P. SEGONDS, éd. bilingue grec-français. Paris, Les Belles Lettres, 1985 et $1986.13,2 \times 20$, CXLVIII + p. $1-210$ et p. $212-474$, index ("Universités de France").

Relire les œuvres de Proclus dans les nouveaux volumes des éditions Budé - La Théologie platonicienne d'abord, par H.-D. Saffrey et L.G. Westerink, puis les Trois études sur la providence, par Daniel Isaac et maintenant l'In Alcibiadem d'Alain Segonds -, c'est comme retourner voir les fresques de Giotto à Assise ou même la chapelle Sixtine après leur restauration. On apprécie les nombreux détails qui étaient auparavant masqués et, entre ceux qui étaient visibles, on voit désormais des liens se tisser. On ne peut qu'en souhaiter autant, en particulier pour l'In Parmenidem (déjà commencé par Carlos Steel et Alain Segonds) et pour le reste de la Théologie platonicienne.

L'édition actuelle comprend deux volumes. L'un couvre le commentaire de $103 \mathrm{~A}$ à $105 \mathrm{~B}$, l'autre le commentaire de $105 \mathrm{~B}$ à $116 \mathrm{~B}$; à cet endroit, le texte 
s'interrompt, incomplet. Comme on peut l'attendre de cette collection, le premier volume contient une importante introduction. Elle est divisée en deux grands chapitres : I, "La tradition de l'Alcibiade ", et II, "Les témoins du texte ", tous deux du plus vif intérêt. Le premier chapitre, après une présentation sommaire de la vie de Proclus, esquisse l'histoire de l'exégèse de l'Alcibiade, depuis les médio-platoniciens en passant par Jamblique et Proclus lui-même, jusqu'à Damascius et Olympiodore.

Je suis d'accord avec Segonds (et lui avec moi) pour penser qu'il n'est pas pertinent de considérer que les références à Jamblique des Discours VI et VII de Julien puissent constituer les fragments d'un commentaire de l'Alcibiade par Jamblique - une idée bien trop avantageuse d'Asmus. Même le seul passage que Dalsgaard-Larsen intègre dans son recueil (comme le fr. 159) ne mérite pas, à mon sens, d'être retenu. D'un autre côté, je suis convaincu que, en maints endroits, les idées de Jamblique, relayées par Syrianus, traversent le commentaire de Proclus. Mais il n'y a aucun moyen d'isoler ce qui appartient en propre à Jamblique.

Avec l'Alcibiade, nous possédons encore, situation exceptionnelle pour les dialogues platoniciens, deux commentaires néo-platoniciens. Segonds, par conséquent, compare longuement Proclus et Olympiodore et montre que, bien qu'Olympiodore ait étudié Proclus, sa lecture du texte de Platon reste tout à fait personnelle. Que peut-on en tirer sur les rapports de Proclus avec Jamblique? Pas grand-chose, à mon avis. Ils sont d'une autre nature. Pour autant qu'on puisse en juger d'après d'autres sources, Proclus fonde son exégèse sur celle de Syrianus qui, lui, travaille à partir de Jamblique et, à l'occasion, le confronte à Porphyre. Dans l'ensemble, Olympiodore simplifie Proclus mais suit sa propre version.

Dans le chapitre II, l'examen de la tradition manuscrite est admirable, beaucoup plus lisible en outre que ne le sont habituellement ces parties. Prolongeant le travail d'Harlfinger, Segonds est capable, en effet, de reconnaître dans le copiste du manuscrit de base Neapolitanus gr. 339 (N) l'humaniste byzantin du XIII ${ }^{e}$ siècle, George Pachymère, ce qui n'est pas sans conséquences biographiques intéressantes.

Le texte et la traduction sont excellents; l'établissement du premier, bien sûr, est largement redevable à l'édition précédente de L. G. Westerink. Je n'ai trouvé que quelques points de détail à critiquer : par exemple, laddition de Westerink (ou $\delta \grave{\varepsilon} \delta \eta \mu t o u \rho \gamma(\kappa \tilde{\omega} \varsigma$ ) en 52, 9 Creuzer est sûrement nécessaire et

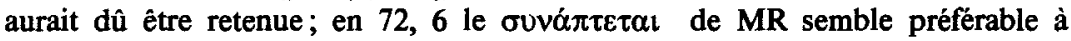

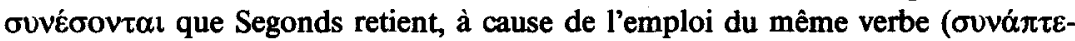

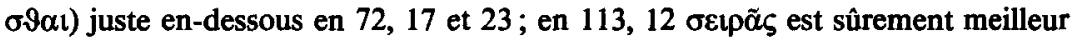

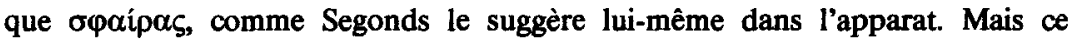
sont des bagatelles. Nous avons désormais à notre disposition un texte de premier ordre.

C'est la lecture des notes qui a été pour moi la plus satisfaisante et la plus instructive. Segonds y expose en détail les parallèles de langue et de doctrine entre l'Alcibiade et le reste de l'œuvre de Proclus, ce qui est très utile. Assurément, j'aurais aimé avoir eu cette édition à ma disposition quand je rédigeais les notes 
de ma propre traduction de l'In Parmenidem. Elle m'eût été d'un grand profit '

A partir du moment où l'Alcibiade est devenu le premier d'une série de dix dialogues qui devaient conduire l'étudiant à une vision complète de la philosophie platonicienne avant d'aborder les deux "sommets", le Timée et le Parménide, tout commentaire de ce dialogue, surtout après Jamblique, devait prendre en compte une bonne part d'exégèse. Il fallait en passer par différents préliminaires et discussions d'ordre général portant sur l'objet de l'enquête philosophique en général et sur la forme du dialogue. Proclus s'y livre dans les règles. La conséquence en est qu'après quatre-vingt-cinq pages Creuzer nous en sommes encore à la première phrase du dialogue et qu'à la page 120 nous n'avons pas fini le discours introductif de Socrate (103 A-104C). Quoi qu'il en soit, ces pages, que Segonds annote toutes avec une admirable minutie, sont d'une grande richesse. J'en recense les sujets principaux, pour les lecteurs auxquels cette cuvre n'est pas familière :

1. le premier principe de la philosophie - la connaissance de soi (qui est aussi l'objet du dialogue), p. 1-10;

2. la priorité de l'Alcibiade dans l'étude de Platon; les parties principales du dialogue, p. 11-18;

3. les différents types d'Amour, p. 30-37, et Socrate comme l'amant divin, p. $38-56$;

4. la nature " démonique » de Socrate, p. 60-67, qui enchaîne sur

5. un discours d'ordre général sur les démons, p. 68-78, qui enchaîne à son tour sur

6. un discours sur le démon de Socrate, p. 78-85 (qui mériterait d'être comparé avec d'autres traitements célèbres de ce thème, tels ceux de Plutarque et d'Apulée).

Cette seule section, on s'en apercevra, renferme une bonne part de la philosophie néo-platonicienne ultérieure et la suite du commentaire en réserve encore plus. Là-dessus, les remarques de Segonds et la série de parallèles qu'il établit sont bienvenues.

J'éviterai d'entrer ici dans la discussion des points de détail. Je voudrais toutefois en relever deux, qui se trouvent m'intéresser particulièrement en ce moment. A leur propos, Segonds fait d'utiles remarques, sans résoudre cependant tout à fait la difficulté que soulève chacun d'eux :

1. En 77, 12, Proclus cite Phaedr. 247 C7-8 sans le vō après $\theta \varepsilon \alpha \tau \dot{\eta}$, comme il le fait également dans In Parm. 1128, 1, et dans Theol. plat. IV 13, p. 4B, 1S.W. De la même façon, Hermias (citant Jamblique). Simplicius et Damascius passent ce $v \tilde{\omega}$ sous silence. Sans parti pris aucun, il semble clair qu'il ne se trouvait pas dans les manuscrits à leur disposition. Jamblique, du moins, fournit une exégèse du passage qui serait tout à fait impossible si ce vã était présent. J'aurais tendance à considérer que c'est une glose byzantine puisqu'elle se comprend facilement (à moins qu'on n'ait un esprit à la Jamblique).

1. Voir Proclus' Commentay on Plato's 'Parmenides', transl. by Glenn R. MorRow and John M. Dillon with Introduction and Notes by John M. DilloN, Princeton, Princeton University Press, 1987. 
2. En 121, 22, lorsqu'il discute du caractère opportun du moment choisi par Socrate pour aborder Alcibiade, Proclus fait remarquer que les pythagoriciens avaient l'habitude d'appeler la Première Cause, celle qui transmet le bien à toutes choses, Kairos, parce qu'elle leur confère la perfection. Il livre aussi cet élément de la tradition dans In Parm. VII 1216, 28 sq., au sein d'un contexte que j'ai qualifié de porphyréen (il critique un commentateur antérieur, en qui j'ai identifié Porphyre, pour avoir adopté cette épithète pythagoricienne du Premier Principe). Segonds écarte avec raison toute référence à une littérature antérieure comme hors de propos (Aristote, de fait, nous apprend dans Пepi Пu $9 \alpha \gamma o \rho \varepsilon i \omega v$ fr. 13 Ross, que les pythagoriciens appelaient le nombre 7 Kairos), mais, pas plus que moi, il ne peut suggérer d'autres rapprochements qui soient pleinement justifiés. Il s'agit là d'un élément intéressant de la tradition pythagoricienne et il serait bon de savoir d'où il vient.

John M. Dillon.

J. L. ACKrill, A New Aristotle Reader. Oxford, Clarendon Press, 1987. $14,5 \times 22,3$, XIII +580 p.

MADE IN OXFORD

J. L. Ackrill, professeur d'histoire de la philosophie à Oxford et grand aristotélicien, capable à la fois d'introduire tout un chacun à Aristote (Aristotle the Philosopher, O.U.P., 1981), et de traduire avec la dernière précision des textes aussi délicats que les Catégories ou le Traité sur l'interprétation (O.U.P., 1963), propose un nouveau recueil de textes choisis d'Aristote, dans le but explicite de faciliter le travail des étudiants de philosophie, voire celui de leurs professeurs. Il opère donc une sélection dans le corpus, d'abord, afin d'extraire, sans trop défigurer le style et la manière de penser, ce qui lui paraît le plus représentatif de la philosophie du Stagirite dans la diversité de ses thématiques e $i$ de ses tendances. Puis une sélection parmi les traductions déjà existantes, afin que le non-helléniste soit mis autant que possible « en position de lecteur du grec" (XII). Enfin, sélection plus drastique encore, il propose une liste thématique de 46 "topics " servant d'entrées, parfois croisées, aux textes choisis, et que complètent d'une part l'indication de quelques passages supplémentaires, d'autre part des renvois aux ouvrages de référence (repris en fin de volume) et à quelques articles mieux ciblés.

La contraction de texte est un exercice scolaire qui a son utilité ; il s'agit ici, pour le meilleur et pour le pire, d'un exercice de contraction de philosophie. Ackrill en connaît parfaitement les dangers, puisqu'il signale lui-même que les listes finales de thèmes, d'articles, d'ouvrages sont « very selective », au point que « n'importe quel connaisseur doit être immédiatement en mesure de dresser des listes alternatives d'une égale utilité " (XIII). Il n'ignore pas non plus que certains thèmes sont encore plus réfractaires que d'autres à cette contraction, et cite comme maltraitées la logique formelle, la biologie et la philosophie politique. Mais, par-delà les lois du genre, on peut encore caractériser de facon 
spécifique cet Aristote de (grande) poche : il est, me semble-t-il, entièrement made in Oxford. Les traductions sont "maison" (Clarendon Aristotle series, ou révision de l'Oxford Aristotle translation); $\propto$ sont, il est bien vrai, parfois les seules et souvent les moins mauvaises, en anglais - mais la souplesse inhérente au découpage en morceaux aurait autorisé l'insertion de traductions partielles, travaillées en profondeur et qui figurent dans des ouvrages moins accessibles. " Maison " aussi, dans leur grande majorité, les ouvrages sélectionnés en référence : un Français peut s'étonner sans chauvinisme, s'il s'agit d'introduire au système et à la problématique aristotélicienne, qu'Aubenque $P$. soit signalé exclusivement comme auteur d'Etudes sur la Métaphysique d'Aristote (Paris, 1979). "Maison " enfin, plus subtilement, le motif des choix, qui profite de l'ordonnance traditionnelle du corpus pour omettre entièrement, avec la fin de la Poétique, tout extrait de la Rhétorique. Si la philosophie du langage ne fait pas partie, et pour cause, des thèmes sacrifiés, on constate cependant qu'elle est restreinte à sa perception analytique, et qu'un lecteur ignorant ignorera toujours qu'Aristote fit aussi une théorie de la métaphore sur laquelle, du Moyen Age à nos jours, tout un pan de la réflexion philosophique et littéraire a cru bon de s'adosser.

Enfin, c'est au sein de cette philosophie du langage restreinte, elle-même, que certains choix me paraissent contestables. Ainsi, il y a, comme on doit s'y attendre, une entrée meaning (p. 569); mais le lieu précis, parfaitement localisable, où Aristote définit explicitement ce que veut dire semainein $t i$, "signifier quelque chose", n'est ni retenu parmi les extraits (on y saute au contraire de Métaphysique IV, 4, 1006a28, à 4, 1008b1), ni même signalé entre crochets de complément. Tel est sans doute le revers de la médaille oxonienne.

Barbara CASsin.

Bruno Pinchard, Métaphysique et sémantique. Autour de Cajetan. Étude et traduction du De Nominum Analogia. Paris, Vrin, 1987. $16 \times 24,188$ p. ( Philologie et mercure »).

Cette édition, avec traduction française et commentaire, du De Nominum Analogia de Cajetan est précédée d'une substantielle introduction d'une centaine de pages qui vise au moins deux buts. Il s'agit d'abord pour B. Pinchard de situer la doctrine cajetanienne de l'analogie par rapport aux doctrines antérieures (Aristote, Thomas d'Aquin, Occam, Duns Scot...), contemporaines (Dominique de Soto, Melchior Cano...) ou postérieures (Suarez, Jean de Saint-Thomas...). Il le fait avec une érudition éblouissante et une densité qui risquent, certes, d'avoir comme effet pervers de rendre cette étude d'un abord difficile au nonspécialiste; mais le texte de B. Pinchard mérite la peine qu'il exige de nous. Il s'agit aussi, et peut-être surtout, d'essayer de penser la catégorie elle-même de scolastique à travers l'exemple à un double titre remarquable de Cajetan. Comme homme du tournant des $x v^{e}$ et $x^{e}{ }^{e}$ siècles il semble, en effet, 
historiquement hors scolastique. Mais il n'est, de ce fait même, pas de meilleur exemple pour illustrer la thèse de $B$. Pinchard selon laquelle la scolastique est « une forme douée d'une consistance propre ", ce qui en fait une figure du savoir qui, même si elle est aujourd'hui pur objet historique, n'en a pas moins pu accueillir des manières d'interpréter un donné théologico-métaphysique qui, en leur temps, ont pu paraître absolument extérieures les unes aux autres. Or le commentateur Cajetan peut, mieux qu'un autre, permettre de vérifier si, à son époque, la scolastique fonctionne ou si elle ne fait que survivre.

Ajoutons que la figure même du cardinal-philosophe, subtil homme d'appareil dont on a pensé faire un pape, rapidement mais justement campée par B. Pinchard, est sans doute irremplaçable pour qui veut saisir, autrement qu'à travers des caricatures, l'autre de la Renaissance humaniste. Quant au problème de l'analogie, "intermédiaire entre la pure équivocité et l'univocité ", sa particularité n'est qu'un trompe-l'œil : c'est bien vers les conditions d'existence et le statut de la métaphysique qu'il conduit nécessairement.

Pierte Pellegrin.

Diderot Studies. T. XXII. Ed. by Otis Fellows and Diana GuIRAGossian CarR. Genève, Droz, 1986. 15,2 × 22,2, 222 p.

Il semble que les Diderot Studies connaissent de réelles difficultés et il est vraiment triste qu'une publication à laquelle ont collaboré des chercheurs aussi éminents que R. Mauzi, O. Fellows, H. Dieckmann, J. Proust ou bien encore J.R. Loy, qui vient de mourir et auquel ce numéro est dédié, se retrouve dans une telle situation.

L'ère semble être révolue des grands questionnements, des polémiques passionnées, des hypothèses à tỏut va, des vastes généralisations séduisantes et parfois trop rapides. Désormais, on défriche chaque centimètre, chaque millimètre devrait-on dire, de l'œuvre de Diderot; tel est le cas de D.J. Adams qui s'intéresse à une édition anglaise des Bijoux indiscrets en vue, on l'espère, d'une étude sur l'influence de Diderot en Angleterre comparable à celle de R. Mortier sur Diderot et l'Allemagne.

Même défrichement serré dans l'article de M.P. MoClymonds et W.E. Rex où les auteurs essayent de repérer, dans la partition des Lamentations de Niccolò Jommelli, les passages auxquels Diderot, dans Le Neveu de Rameau, pourrait faire référence. Ce qui aboutit à une analyse de la Nature et de son dénaturement dans l'œuvre de Diderot, d'où il ressort que chaque niveau d'illusion, comme un jeu complexe de miroir, renforce en fait l'impression de vérité de l'ensemble.

A. Burns et J. Garagnon ont, tous deux, le premier pour un article de l'Encyclopédie, le second pour un passage de Jacques le fataliste, tenté le même type d'approche, à savoir de retrouver les sources, "l'infra texte ", et d'établir de nouveaux rapports afin de mieux cerner la maturation de la pensée de Diderot et son élan créateur. A. Burns démontre ainsi que S. Formey est à la base 
de l'article « Juste » de l'Encyclopédie, et non F.H. Strube de Piermont comme le pensait le professeur Thielemann. Peut-être Burns aurait-il pu également, une fois les filiations établies, s'intéresser à cette communauté de pensée des trois textes. J. Garagnon, effectuant un double rapprochement entre le Supplément et Jacques, renforce l'idée qu'il existe aussi une continuité de réflexion très nette entre les deux cuvres, qui s'ajoute à celle déjà décelée entre les contes et Jacques. D'ailleurs, ne peut-on penser que Jacques, roman sur les paysans ("Un Jacques est un homme...") est aussi le roman de l'Autre, comme le Supplément est l'expression de la philosophie de l'Autre? Le paysan n'est-il pas, pour le philosophe des Lumières, un "bon sauvage ", en quelque sorte, l'égal d'Orou?

L'œuvre philosophique de Diderot est elle aussi étudiée dans ce tome XXII, à travers deux articles : "Diderot as Philosopher " de J. Barzun et " Denis Diderot and the Limits to Reason » de A. Davidson. Les deux chercheurs partent sur des pistes identiques en s'interrogeant sur ce que le concept de Nature recouvre dans la pensée de Diderot, ainsi que sur ses prolongements politiques. Ils en arrivent à une conclusion assez similaire : le philosophe n'était pas un matérialiste. Il serait même un phénoménologiste avant la lettre pour Barzun qui tente malgré tout de le replacer dans le contexte scientifique de l'époque, pour finalement affirmer qu'il était en avance d'au moins un siècle. Quant à Davidson, il analyse la complexité des idées diderotiennes sur le réel pour se demander si l'art n'était pas au centre de la problématique des Lumières tant Diderot attachait d'importance à la recherche de nouvelles formes utilisant jusqu'à la fable, pourtant condamnée par certains penseurs de l'Encyclopédie, comme porteuse de superstition.

Avec ce supplément $n^{\circ}$ 2, le premier étant dans le tome précédent des Diderot Studies, F. A. Spear poursuit son travail bibliographique sur Diderot, en mettant à jour son répertoire paru en 1980, mais dont les références s'arrêtaient aux livres publiés avant 1975 .

Quel jugement chaque époque porte-t-elle sur les « grands écrivains fondateurs d'idéologie " ? C'est la question à laquelle $\mathbf{R}$. Trousson tente de répondre en s'intéressant aux réactions de la presse face à la commémoration du bicentenaire de la naissance de Diderot en 1913. Symbole pour les uns de l'anticléricalisme et de la Révolution française, il devient pour les autres un anarchiste (le souvenir de Bonnot est encore tout proche), un athée, un cosmopolite (la proximité de la guerre se fait ici sentir). Au total, les joumaux se polarisent autour de deux idées-forces : la filiation Lumières-Révolution et l'amalgame, dénoncé ou revendiqué, entre les républicains modemes et les philosophes des Lumières.

Partant des analyses de M. Bakhtine, R. Mesavage (« Dialogue and Illusion in Jacques le fataliste ") cherche à apporter un nouvel éclairage sur un sujet souvent étudié (cf. H. Dieckmann) : le dialogue. Son idée est de démontrer comment sens et forme se renforcent en s'interpénétrant. A une tentative philosophique qui vise à retenir le jugement, à le complexifier, répond une esthétique du dialogue, expression de l'ambiguîté. Mais le véritable intérêt de cet article est d'avoir montré qu'à chaque fois que l'on est en présence d'un discours à sens unique, dogmatique et interprétatif, on passe, comme pour mieux souligner leur "irréalité ", d'une tournure réaliste (qui colle à la réalité des 
mots) à une narration fondée sur un rythme poétique, une sorte de ritournelle, symbolisée par la répétition des "c'est écrit là-haut».

Allant de la grivoiserie avec les Bijoux indiscrets et certaines historiettes de Jacques, à l'étude des comportements sexuels déviants dans la Religieuse, en passant par une théorisation des rapports de couple au sein du mariage dans le Supplément, l'œuvre de Diderot est traversée par de constantes réflexions sur le thème de la sexualité. A. Parker ( $"$ Did/erotica : Diderot's Contribution to the History of Sexuality ") envisage d'analyser la vision diderotienne de la sexualité féminine. Dès lors, on comprend à la lecture de cet article, que le philosophe n'a jamais pu résoudre un double dilemme : l'un d'ordre psychophysiologique, comment vivre en bonne intelligence avec des femmes dont on ne peut se passer mais dont les bizarreries sont liées à leur sexualité ? et l'autre d'ordre sociohistorique, quelle que soit l'injustice de son sort, la femme doit se soumettre au mariage et à l'opinion publique. Pour A. Parker, la principale contribution de Diderot à l'histoire de la sexualité est d'avoir montré qu'il n'y avait pas d'esprit sans corps, ni de métaphysique sans Eros, en rattachant chaque individu à l'espèce et chaque espèce à la nature.

Enfin, J. Undank (" Diderot and the Phenomenology of the Ordinary ") met l'accent sur un aspect peu étudié de l'œuvre de Diderot : son théâtre. Pour comprendre l'échec de ce théâtre, le pourquoi de cette désuétude, il faut partir d'une réflexion sur la dialectique de l'ordinaire et de l'extraordinaire. Diderot met en scène des personnages de la vie quotidienne. Mais comment traduire esthétiquement l'ordinaire si ce n'est par le biais d'une forme déjà connue et expérimentée, palpable par le spectateur? C'est pourquoi il choisit de se rapprocher des ressorts classiques, pitié, terreur, des drames antiques. Ordinaire, extraordinaire, la contradiction ne sera pas résolue...

On le voit donc, ce tome XXII des Diderot Studies presente des approches multiples et renouvelées pour toutes les facettes d'une cuvre immense et riche. Emettons cependant le regret que la perspective historique, le nécessaire déplacement dont parlait $P$. Vilar, soit malgré tout absente de ces diverses contributions.

Carl ADERHold.

Denis Diderot, 1713-1784. Colloque international, Paris-Sèvres-Reims-Langres (4-11 juillet 1984). Actes recueillis par Anne-Marie Choullnet. Paris, Aux Amateurs de livres, 1985. $16 \times 24,551$ p. ("Collection des Mélanges de la Bibliothèque de la Sorbonne $", 8)$.

La particularité du Colloque international Diderot est de s'être déroulé dans quatre lieux successifs : Paris, Sèvres, Reims et Langres. Il s'insère d'ailleurs dans les nombreuses manifestations qui ont jalonné l'Année Diderot (voir 1984. L'Année Diderot, informations recueillies par A.-M. Chouillet avec la coll. de R. Desné et Fr. Dougnac, Paris, P.U.F., 1984). Après la séance inaugurale de 
Paris, le discours du ministre de la Culture, les allocutions de $\mathbf{M}^{\mathrm{me}}$ Ahrweiler, de MM. Pomeau et Ehrard, c'est à Sèvres que les chercheurs se sont réunis pour évoquer tous les aspects de l'œuvre de Diderot.

Cinq communications sont consacrées au Diderot philosophe, une séance organisée par Y. Belaval (5 juillet). P. Casini étudie d'abord la façon dont Diderot s'est créé une image personnelle de l'Antiquité. Dans une communication intitulée « Diderot et Malebranche : de l'amour de l'ordre au goût de l'ordre », J. Deprun montre ensuite que Diderot, dont l'admiration pour Malebranche est bien connue, participe à la laïcisation de l'idée d'ordre et à sa transposition naturaliste. Quant à J. Spink, il analyse comment Diderot se livre à la réhabilitation de l'idée de pitié : car il "veut être bon ". "A côté de la justice, qu'il reconnaît comme la seule vertu [...], il place la bonté comme une qualité morale suprême " (p. 57-58). L'objet de la communication d'A. Thomson est d'étudier la conception de la matière chez Diderot et chez La Mettrie, en laissant de côté « la question très problématique des influences » du second sur le premier (p. 61). Enfin, A. Vartanian souligne que la science moderne, en se livrant à des manipulations génétiques et à des transplantations d'organes, traite les êtres vivants comme des ensembles mécaniques et modifie ainsi la frontière conceptuelle entre ce qui est vivant et ce qui ne l'est pas : ces mutations trouvent une anticipation "précoce mais géniale" (p. 69) dans l'œuvre de Diderot et notamment dans Le Rêve de d'Alembert.

J.-M. Goulemot était l'organisateur de la session de l'après-midi, dédiée à Diderot et l'amour. Ouvrant la séance de travail, G. Benrekassa (" Diderot et l'honnête femme") note que "le problème de l'honnête femme se définit idéologiquement pour Diderot avec une cohérence absolue, et cela sans contradiction autre qu'historique avec une physique de l'amour extrêmement libre " (p. 89), tandis que C. Blum remarque que Diderot - tout comme Rousseau - lie fesser et confesser. Dans la suite, J.-C. Bonnet, après avoir critiqué la méthode biographique qui utilise la correspondance amoureuse «comme un simple document", se livre à une analyse serrée des Lettres à Sophie Volland: « L'Écrit amoureux ou le fou de Sophie. » Il établit en particulier que « véritable laboratoire sémiotique, la correspondance amoureuse a une fonction décisive dans l'invention du principe de l'œuvre" (p. 110). B. Fink, un peu comme A. Vartanian, souligne l'aspect précurseur de l'cuvre de Diderot. Dans les Éléments de physiologie, bien avant Freud, il aurait pressenti que l'ensemble des pulsions inconscientes de l'individu se groupent en deux ensembles opposés, l'Amour et la Faim. C. Lafarge soutient ensuite que la correspondance à Sophie Volland est un «art de vivre » et cherche à éclaircir comment Diderot a aimé. Enfin, B. de Négroni se demande quel est le rôle des femmes dans l'économie générale des dialogues de Diderot.

Le matin du vendredi 6 juillet fut l'occasion d'évoquer le thème de Diderot et la Politique, moment du Colloque organisé par P. Vernière. F. Diaz analyse d'abord dans sa communication les oscillations, les changements, les contradictions de la politique de Diderot. En sa dernière évolution, sa pensée politique, au lieu de l'enfermer dans le renoncement, le porte « sur la ligne même de la pensée la plus avancée des Lumières " (p. 158). Le séjour de Diderot à SaintPétersbourg apparait comme une "expérience majeure ", comme une coupure 
dans l'expérience de Diderot, un passage de l'illusion au désenchantement. Mais G. Dulac montre que cette rupture n'existe pas vraiment car l'essentiel était acquis avant, par une réflexion préalable. G. Goggi, dans Diderot et Médée dépeçant le vieil Eson, analyse la présence et l'origine de cette image présente dans la Correspondance, la Réfutation d'Helvétius et l'Histoire des Deux Indes : cela lui permet de suivre les variations du "pessimisme historique" du philosophe sur la situation française de 1770-1780. Ensuite, M. Skrzypek fait remarquer que, contrairement à Rousseau et à Mably qui ont proposé un projet de réforme politique pour la Pologne, les observations de Diderot sont dispersées dans tous ses ouvrages. Cependant, le philosophe n'est pas un écrivain pro-russe et ses solutions se distinguent par leur radicalisme. Clôturant ce chapitre politique, A. Strugnell intervient sur Diderot et l'idée de Nation : le noyau de sa pensée politique, c'est de considérer qu' «il n'y a point de vrai souverain que la nation; il ne peut y avoir de vrai législateur que le peuple » (p. 197). La nation assure liberté, sécurité, bonheur, dignité à chaque individu qui la compose.

Quelque huit communications traitaient du problème complexe de Diderot et l'invention littéraire (organisateur $\mathrm{E}$. Walter). Dans une communication intitulée "Corps oublié ou la Métamorphose du Nageur", P. Chartier s'intéresse de près à l'image singulière du nageur de la fin des Eléments de physiologie et à la fable du jeune Mexicain emporté par les flots de l'Entretien avec la Maréchale. Selon J.-P. Seguin, l'invention littéraire chez Diderot n'est pas d'abord thématique, car « elle est de l'ordre du style » (p. 219); dans les Eléments de physiologie, il "a porté à son point de perfection un discours fait de mimétisme de la communication orale » (ibid.). H. Cohen analyse ensuite l'influence du Mémoire sur les fabliaux (1746) de Caylus, l'apparition de motifs carnavalesques et d'éléments de la tradition gauloise dans Les Bijoux indiscrets. Puis R. Kempf montre que ces mêmes Bijoux indiscrets sont finalement « une œuvre sévère » qui renvoie aux articles philosophiques de L'Encyclopédie et à la réflexion mathématique de Diderot. Quant à A. Siemek, il se penche sur l'étude des implications idéologiques des traductions des textes de Diderot en polonais. Y. Sumi, de même, traite la question délicate de la traduction des lettres de Diderot à Sophie Volland : il conclut que le japonais « se prête bien au transcodage de la richesse connotative des lettres de Diderot " (p. 260). Enfin, J. Veselý ( " Diderot et la mise en question du roman " réaliste " du XVIr siècle ") montre que Diderot a transformé un genre romanesque qui sert désormais à la quête de la vérité.

Le samedi 7 juillet trois congressistes seulement abordèrent le thème de Diderot et la science, séance organisée par J. Roger. Dans un premier temps, J. Dhombres poursuit un double propos: jalonner la formation mathématique de Diderot et tester la solidité de sa culture scientifique. Puis J. Mayer établit que Diderot s'intéresse aux monstres de façon priviligiée : il s'agit à la fois d'une fascination où se mêlent attirance, horreur et d'une recherche de l'exceptionnel, révélateur des tendances universelles; l'intention finale étant de prendre l'ensemble de la nature comme objet scientifique. Pour finir, R. Rey considère qu'un ouvrage d'un célèbre médecin du XvIIr siècle, La Médecine de l'esprit du $\mathrm{D}^{r}$ Le Camus, 
est comme un "fil d'Ariane" dans un ensemble de questions que se pose Diderot, notamment sur la guérison des maladies mentales et sur la conception de l'homme de génie.

Nombreuses furent les communications concernant Diderot et l'Art, séquence dont l'organisation avait été confiée à J. Chouillet. Dans « Peinture et cruauté chez Diderot", R. Démoris remarque que, volontiers, le philosophe cherche dans «le registre du corps souffrant " une émotion esthétique que la peinture historique et mythologique ne sont plus capables de lui foumir; qu'il ressent une certaine admiration pour le crime " sur la toile et sur le marbre " à condition qu'il soit d'envergure, car "sévérité, vérité, cruauté sont du même bord : celui de la grandeur" (p. 301). Les "Aspects de la pédagogie musicale de Diderot" sont évoqués par B. Didier qui rappelle que le philosophe surveillait les leçons de clavecin données à sa fille; ainsi apparaissent les principes de la pédagogie musicale de Diderot qui est faite du refus de la facilité : on peut même parler d'une pédagogie de la difficulté. Ph. Junod se pose ensuite la question des rapports de Diderot et du peintre Hubert Robert. De son côté, G. May étudie la façon dont les Salons ont joué un rôle essentiel dans l'inspiration littéraire de Diderot qui, vieillissant et fatigué, a pu ainsi retrouver «la fraîcheur d'impression et l'énergie d'expression qu'il croyait avoir irrémédiablement usées au cours de ses décennies de labeur forcené de l'Encyclopédie» (p. 335). Cette importante partie du colloque consacrée à l'esthétique de Diderot se poursuit par une communication de B. Saint-Girons qui, analysant le tableau de Deshays (exposé au Salon de 1763) - La Chasteté de Joseph -, rend compte de la "fascination qu'exercent certains écrits de Diderot " et de la contribution de celui-ci à une théorie du sublime. Enfin, Ph. Stewart souligne, dans " Le rôle de la gravure dans l'cuvre de Diderot ", que le philosophe, en 1765, avait une connaissance approfondie de cette technique d'expression, essentielle pour la diffusion des ceuvres.

Dans la soiree du 7 juillet, J. Starobinski fit une conférence, « Du pied de la favorite au genou de Jacques ", où il étudie les métamorphoses culturelles, érotiques, religieuses du corps humain - en particulier du pied - et analyse le monde des sensations physiques présentes dans l'univers sensoriel du récit : « Raconter, chatouiller, c'est l'excitation simultanée d'un double registre sensoriel » (p. 360). Cette conférence fait la transition avec l'ensemble suivant : Diderot et l'Etranger, organisé par R. Mortier (8 juillet). P. France observe d'abord que Diderot n'est jamais allé en Angleterre et n'a eu de l'anglais qu'une connaissance livresque, puisée dans un dictionnaire anglais-latin. Il analyse le travail de traducteur de Diderot et se livre à quelques réflexions sur le sens de la traduction dans l'œuvre du philosophe. F. Lafarga assure, de son côté, que Diderot était peu connu en Espagne au XVIII siècle; on trouve peu de références à ses ceuvres dans les ouvrages espagnols contemporains, contrairement à Voltaire et à Rousseau. D'ailleurs Diderot, à la différence de ceux-ci, n'a pas vu l'ensemble de son cuvre condamnée par l'Inquisition : quelques ouvrages seulement furent proscrits. A propos du Brésil, L.C. Jobim constate que, de nos jours, l'intérêt pour l'encyclopédiste s'est déplacé du penseur politique (conceptions sur le colonialisme) vers "l'homme de lettres ». Des soixante-cinq articles de l'Encyclopédie sur le Japon, H. Nakagawa distingue ceux écrits à la manière de Jaucourt 
- qui consacrent la supériorité de la civilisation occidentale - de ceux rédigés par Diderot qui tentent d'établir une analogie entre le Japon et la France (p. 420). En R.F.A., observe J. von Stackelberg, aujourd'hui, Diderot a « une place très réduite" (p. 423) dans la vie culturelle, malgré des propagateurs allemands célèbres : Lessing, Schiller, Goethe; il décrit aussi les problèmes de traductions que posent les textes diderotiens, notamment le Neveu de Rameau. K. Schnelle, puur la R.D.A., rappelle l'importance des recherches instituées par W. Krauss dès les années 1948-1949 dans le domaine de la philosophie des Lumières. Cela n'est sans doute pas étranger à l'intérêt très vif qu'inspirent les ouvrages de Diderot, lesquels, en R.D.A., sont « diffusés, parfois à des tirages impressionnants [et] constamment épuisés" (p. 432). T. Voronova fait ensuite une mise au point concernant les manuscrits de Diderot conservés à la Bibliothèque d'Etat de Leningrad. Ils ont été achetés par Catherine II - en même temps que sa bibliothèque - en 1765 et transportés à Saint-Pétersbourg en 1786. Au total, deux mille neuf cent quatre unités. L'auteur décrit six cartons d'inédits utilisés par les éditeurs des Euvres completes. Enfin, comment Diderot est-il perçu en Chine? Guan Shibin note qu'il « fait l'admiration de tous les chercheurs en philosophie" et depuis trente ans on a traduit et publié «une quantité d'ouvrages " de l'encyclopédiste (p. 447).

Les 8-9 juillet, le Colloque Diderot s'est transporté à Reims pour la dernière journée consacrée à Diderot homme de théâtre. La première demi-journée, Diderot dramaturge, fut organisée par $\mathbf{R}$. Desné. Deux représentations théâtrales furent proposées : Feux de la Conversation (mise en scène J. Deloche) et Entretien d'un pere avec ses enfants (Compagnie J. et C. Roche). Dans un premier temps, D. Fletcher s'attache à relever certains éléments picturaux dans les théories dramatiques de Diderot, cependant que H.V. Seifert montre que Sade fut un fervent lecteur du Père de famille: "c'est un chef-d'ouvre", "un code d'instruction pour toutes les mères de famille et c'est vraiment sublime " (lettre à sa femme, 14 juillet 1780) (voir p. 471-472). " Diderot et la leçon du théâtre antique ": tel est le titre de l'intervention de $\mathbf{R}$. Trousson. Diderot ne semble retenir du théâtre grec que les scènes violentes exprimant la "nature " et le " vrai ". Lorsqu'on reproduira « les effets terribles » de ce théâtre, pense-t-il, on aura retrouvé la fonction première du théâtre. Aussi l'essentiel des remarques de Diderot portera sur les moyens utilisés par les Anciens pour produire ces effets, en particulier la pantomime à laquelle une "place considérable " est réservée (p. 482). P. Zaborov, de son côté, constate que les idées réformatrices de Diderot en matière théâtrale ont éveillé un grand intérêt dans la Russie du $\mathrm{XVIII}^{\mathrm{e}}$ siècle; S. Glébov traduit Le Père de famille qui sera représenté par des acteurs français dès le début des années 1770 .

La seconde demi-journée orientée vers le théâtre - Le théatre d'aujourd'hui devant Diderot - était placée sous la responsabilité de J. Darolles et de J. Deloche. Dans sa conférence consacrée aux canevas dramatiques de Diderot, J. Chouillet observe que les années 1757-1759 sont des années d'intense réflexion dramatique : des ébauches sont conçues dans l'intention de réformer le théâtre. Diderot pensait qu'il fallait adapter à la scène française les recettes qui ont permis de rénover le théâtre anglais. En acclimatant Shakespeare, on parviendrait à " la tragédie domestique " dont Diderot attendait des effets identiques à ceux 
provoqués par la tragédie antique. Il reste que ces ébauches dramatiques pourraient fort bien se transformer en pièces à deux conditions que J. Chouillet énumère (p. 515). Enfin, un débat a réuni plusieurs professionnels du théâtre sur le thème: "Mettre en scène Diderot aujourd'hui." "

Le Colloque Diderot s'est terminé à Langres les 10-12 juillet 1984 par un concert donné par les musiciens des Arts florissants, par une visite à l'exposition du Musée du Breuil St. Germain, "Diderot et la critique de salon 1759-1781 " (voir le catalogue), enfin par une représentation théâtrale, Le Philosophe amoureux (dramaturgie de G. Peltier).

On le voit, par ces quarante-huit communications, $\propto$ Colloque international Diderot - parfaitement réussi - fut un moment essentiel des manifestations qui ont émaillé l'Année Diderot. Il faut rendre hommage aux organisateurs d'un si grand et si long travail. Il faut aussi remercier $\mathbf{M}^{\mathrm{me}}$ Chouillet qui s'est chargée de la tâche - difficile - de recueillir les Actes de ce colloque et de les publier rapidement : il est bon qu'un délai aussi court que possible sépare la tenue du colloque lui-même de la publication des actes.

Tel quel, le Colloque Diderot est une mise au point, une somme critique sur l'œuvre protéiforme du philosophe, une bonne image des différents courants actuels de la recherche. D'ailleurs, certains domaines sont très étudiés avec sept ou huit communications $(14,5 \%$ et $16,6 \%$ du total) - Did. et l'amour, Did. et l'invention littéraire, Did. et l'étranger -, alors que d'autres - Did. et la science - paraissent délaissés (trois interventions : 6,2 \%) : nous manquons d'historiens des sciences. Dans l'ensemble, l'ouvrage est bien présenté : il est pourvu d'un index et d'abondantes illustrations. Il faut seulement déplorer, au moins dans l'exemplaire du service de presse, une erreur de composition typographique (p. 398-399) ainsi que quelques coquilles ici ou là. Disons enfin que, en couverture intérieure, on signale l'existence de la Société Diderot, fondée en octobre 1985 et présidée par J. Chouillet, qui édite la revue Recherches sur Diderot et l'Encyclopédie.

Hervé GuENot.

Maine de Biran, Euvres. Sous la dir. de François Azouvi. Vol. II : Mémoires sur l'influence de l'habitude sur la faculté de penser. Ed. par Gilbert Romeyer-Dherbey. Paris, Vrin, 1987. $14 \times 21, \mathrm{XX}+408$ p. (« Bibliothèque des textes philosophiques $")$.

Le mémoire sur l'Influence de l'habitude sur la faculté de penser est un texte si célèbre qu'il n'est pas besoin de le présenter. Mais il importe de signaler la remarquable édition critique et diplomatique que vient d'en faire paraitre Gilbert Romeyer-Dherbey dans le cadre de la réédition des Euvres de Maine de Biran, patronnée par le C.N.R.S.

Dans son introduction, il retrace d'abord, comme il sied à tout éditeur, l'histoire de ce mémoire qui a connu deux rédactions successives plusieurs fois 
remaniées l'une et l'autre. Cet ouvrage, le seul achevé et publié par l'auteur, n'échappe donc pas à la règle commune de tous ses autres écrits, et c'est ce qui amène Gilbert Romeyer-Dherbey à s'interroger sur le pourquoi de ces remaniements perpétuels. Aussi son introduction se transforme-t-elle ensuite en un brillant essai sur les raisons qui peuvent permettre d'expliquer pourquoi Maine de Biran n'a cessé de revenir sur ce qu'il a écrit, sans jamais parvenir à produire l'œuvre définitive qu'il a cependant tenté de réaliser à travers les différents livres qu'il a laissé inachevés. Plutôt que d'en chercher la raison principale dans son caractère et le décousu de sa pensée, il tente de montrer que cette perpétuelle remise en question est due au fait que cet auteur, en philosophe authentique, a toujours été attentif à l'existence du moi, qui ne se livre jamais complètement d'un seul coup au monologue intérieur.

A cette introduction, il a ajouté un avertissement dans lequel il détaille le contenu de ce volume, à savoir : 1) les introductions inédites à l'ébauche et au brouillon de la première rédaction; 2) le texte entier inédit aussi de celle-ci, qui date de 1800 , suivi des notes marginales de l'auteur; 3) le texte de la seconde rédaction publiée par Maine de Biran en 1802, puis par Cousin en 1841 et par Tisserand en 1922 (réédition en 1954); 4) les notes ajoutées par Biran en marge de son exemplaire du texte imprimé, recueillies déjà par Tisserand, et sur les feuilles inédites qu'il y a insérées, celles qu'il a écrites dans les marges du livre de Dutrochet sur l'habitude; 5) une série de quatre appendices contenant respectivement : le rapport de Destutt de Tracy sur le mémoire, le compte rendu qu'en a fait Degérando, celui de Cabanis, une étude anonyme inédite sur ses deux rédactions, due sans doute à Cousin. Dans cet avertissement, Gilbert Romeyer-Dherbey explique aussi les sigles, plus nombreux que dans les volumes précédemment parus, qui renvoient aux notes que l'auteur a inscrites dans les marges du manuscrit de la première rédaction, à celles du livre imprimé, à celles encore qu'il a ajoutées dans les marges de celui-ci, aux variantes, et à ses propres notes d'éditeur.

Celles-ci, très précises et très utiles, sont pour la plupart d'ordre historique : il y identifie les auteurs auxquels Biran fait allusion, cite leurs textes quand besoin est, précise les références à leurs œuvres ou les rectifie, éclaire le sens de certains termes.

Sous le titre "Bibliographie ", il dresse l'état des différentes éditions du mémoire, des ouvrages qui font partie de sa postérité, des études qui en développent certains points et de celles qui l'analysent.

Le tout est complété par un index des noms cités tant par l'éditeur que par l'auteur, et par un index des matières.

C'est dire la qualité et l'intérêt de cette nouvelle édition d'un des textes les plus importants de Maine de Biran.

Jean ÉcoLE.

Bernard BourgeoIs, Le Droit naturel de Hegel (1802-1803). Commentaire: contribution à l'étude de la genèse de la spéculation hégélienne à Iéna. 
Paris, Vrin, 1986. $14 \times 22,664$ p. (« Bibliothèque d'histoire de la philosophie »).

Le livre porte en sous-titre: "Contribution à l'étude de la genèse de la spéculation hégélienne à Iéna. " De fait, le "commentaire " de l'article du Journal critique de la philosophie, traduit par B. Bourgeois et paru chez le même éditeur sous le titre " Des manières de traiter scientifiquement du droit naturel » (Paris, Vrin, 1972, 104 p.), constitue une contribution fondamentale à l'étude de la pensée philosophique de Hegel à Iéna.

Le texte de l'article «constitue assurément, écrit B. Bourgeois, un point nodal essentiel » et même «décisif, sur la ligne du développement de l'cuvre de Hegel " (p. 7) - et ceci pour deux raisons : d'abord, parce que ce dernier produit de l'entreprise critique commune de Schelling et de Hegel marque le moment où "le discours critique [...] se nie comme tel en déployant comme son contenu même l'Idée spéculative " (p. 7); ensuite, parce que la systématisation spéculative-rationnelle de l'article sur le droit naturel "recueille tout l'héritage des méditations du jeune Hegel, de Tubingen à Francfort, orientées, comme on sait, sur les questions théologico-politiques" (p. 7-8). Ainsi le commentaire fera-t-il appel à tous les textes antérieurs, publiés et non publiés par Hegel, et au manuscrit du Système de la vie éthique (p. 17-18 et notes). Le caractère d' " ceuvre germinatrice " (p. 8) du texte commenté conduira enfin B. Bourgeois à évoquer, dans la conclusion de l'ouvrage, le " destin " de l'article sur le droit naturel (p. 631-639). Dans le manuscrit sur la Logique, métaphysique et philosophie de la nature de 1804-1805 se produit la «novation " qui consiste "d'un même mouvement, à spiritualiser l'absolu et à absolutiser la dialectique logique " (p. 633) ; dans le manuscrit de 1805-1806 sur la Philosophie de l'Esprit apparait le «principe supérieur des temps modernes »: le principe de la subjectivité libre (trad. G. Planty-Bonjour, Paris, P.U.F., 1982, p. 95) - principe "ignoré » par Platon; à partir de la Phénoménologie de l'Esprit et jusqu'aux Principes de la philosophie du droit de 1821 « se concrétise la promotion pratique et théorique du concept ou du soi comme autoposition, détermination ou différenciation " (p. 636). La philosophie hégélienne du droit est essentiellement interprétée comme philosophie de la liberté.

Est-ce là un simple "retour » aux principes du droit naturel moderne que l'article sur le droit naturel avait critiqués? Le dépassement hégélien de la théorie " moderne " (kantienne et fichtéenne) de la liberté doit être compris à partir de la notion d'Esprit; le niveau éthique n'est plus compris comme nature, puissance éthique naturelle opposée à l'abstraction du droit moderne, mais comme Esprit objectif, lieu de «l'activité dialectique de l'Esprit » (p. 638) et comme tel, d'ailleurs, relativisé, "surpassé comme totalisation de soi de l'Esprit par l'art, la religion et la philosophie " (p, 639).

Les derniers développements du commentaire permettent de situer le sens de l'interprétation qui est proposée de l'article sur le droit naturel : ce texte présente "l'intuition fondamentale du concept selon lequel, en celui-là, [i.e. le hégélianisme] se structureront le droit et l'Etat " (p. 639). Pour B. Bourgeois la philosophie hégélienne est une philosophie de la liberté en tant qu'elle est une philosophie du concept : c'est $c e$ qu'il a longuement montré dans un article 
intitulé "Dialectique et structure dans la philosophie de Hegel" (Revue internationale de philosophie, 1982). Rappelant l'expression de l'Encyclopédie : «Le concept est ce qui est libre " $(\S 160)$, il ajoutait : "C'est bien l'un des plus beaux thèmes hégéliens que celui suivant lequel " seul ce qui est libre peut se donner ses déterminations comme quelque chose de libre, les laisser-aller comme quelque chose de libre" (Philosophie de la Religion, "Les religions de l'individualité spirituelle », trad. Gibelin, Vrin, 1972, II, 2, p. 57) : être libre, c'est libérer " (art. cit. p. 173). On comprend ainsi le sens même de l'entreprise du commentaire - tel qu'il est énoncé dans la phrase de conclusion : "... le développement ultérieur de la philosophie hégélienne du droit, à travers tous ses enrichissements, ne sera, en vérité, que la conceptualisation du contenu éthique intuitivement structuré, d'abord offert par le génial propos de l'essai sur le droit naturel " (p. 639).

Le propos de B. Bourgeois est donc de suivre pas à pas la démarche extrêmement ramassée de l'article sur le droit naturel, et d'abord à partir des articulations soulignées par les premiers éditeurs des œuvres complètes de Hegel ; le texte est ainsi découpé en quatre parties précédées d'une introduction : les quatre grandes parties du commentaire suivent les quatre parties du texte. Si les deux premières parties - critique des conceptions empiriste puis formaliste du droit naturel - doivent être déjà comprises par rapport à la manifestation encore insuffisante de l'absolu (« absolutisations fautives de moments relatifs vrais de cet absolu ", p. 21), c'est dans la troisième partie du texte que l'on se trouve en présence du " développement spéculatif de la manifestation éthique de l'absolu » (ibid.). Cette partie de l'article est à comprendre comme "foyer privilégié de toute la pensée - passée, présente et future - de Hegel " (ibid.). La quatrième partie du texte fait apparaître l'enracinement de la philosophie dans le développement de l'histoire éthico-politique - B. Bourgeois insiste dans son commentaire sur une des conséquences les plus éclairantes de cette analyse : la découverte du lien de la philosophie au devenir de la raison absolue relativise le procédé critique encore appliqué au début de l'article à l'encontre du formalisme kantien-fichtéen à qui on a reproché de ne pas réaliser "dans son contenu l'universalité et vérité » qu'il prétend posséder (p. 623). " Rejetant également la fixation du passé et l'absolutisation d'un présent qui s'abstrait de lui-même comme héritier du passé, la philosophie rationnelle adhère à l'advenir présent du futur comme réalisation objective de la raison qu'elle sait lire en elle-même » (ibid.).

Il y a donc dépassement de la problématique de jeunesse concernant la positivité et accès à la problématique maitrisée de l'histoire; ceci n'a été rendu possible que par la découverte du "sacrifice de l'absolu », représenté dans la " tragédie de l'éthique » et qui a constitué le thème central de la troisième partie de l'article.

Une partie considérable du commentaire est consacrée à l'analyse de ces pages célèbres et énigmatiques. Ainsi s'éclaire le double enjeu de l'interprétation : la restitution, d'une part, de leur double horizon historique de signification : l'histoire de la philosophie grecque et la réflexion moderne sur la culture grecque - art et philosophie; et, d'autre part, la mise à jour de l'émergence de la pensée originale de Hegel : l'accès de cette pensée au stade proprement hégélien 
de la spéculation. On peut se référer, en particulier, à trois moments de l'analyse : la confrontation, en premier lieu, de la conception hégélienne de la tragédie, avec sa "triple signification, onto-théologique, éthico-politique et artistique" (p. 459), à la conception schellingienne du tragique (p. 453-476); « nous avons affirmé, note B. Bourgeois, que l'exposition [...] de l'absolu lui-même comme tragédie, constituait une novation décisive dans l'élaboration de la philosophie spéculative hégélienne " (p. 460); l'analyse, en second lieu, de l'émancipation "du prestige schellingien" au niveau de "l'affirmation décisive que "l'esprit est plus haut que la nature" "(p. 521-524); l'examen, en troisième lieu, du rapport du droit à la morale et du thème de l'éducation (p. 524-554).

La « thématique ontologique » (p. 627) de la troisième partie de l'article est actualisée dans la quatrième partie du texte au niveau du devenir de la vie éthique et de son accès historique au niveau de l'État moderne - État qui est « la réalisation éthique de l'identité rationnelle originaire de l'identité à soi ou positivité (au sens positif du terme) et de la différenciation d'avec soi ou négativité " (p. 627-628). Une étape décisive est franchie avec le texte de 18021803 au niveau de " la négation rationnelle concrète de l'abstraite opposition du droit naturel et du droit positif, c'est-à-dire de toute la problématique antérieure du droit naturel moderne » (p. 629).

Tout au long des analyses claires et profondes qui constituent le commentaire de B. Bourgeois, l'interrogation philosophique est maintenue à son plus haut niveau et l'on pense à la conclusion de l'essai sur La Question de la technique de Heidegger : "Car l'interrogation est la piété de la pensée."

Marie-Jeanne KÖNIGSON-MONTAIN.

Solange Mercier-Josa, Retour sur le jeune Marx. Deux études sur le rapport de Marx à Hegel. Paris, Méridiens Klincksieck, 1986. $16 \times 23,195$ p. (« Philosophie ").

Retour sur le jeune Marx de Solange Mercier-Josa reste fidèle au défi lancé dans son précédent ouvrage à l'interprétation althussérienne du " renversement " marxien comme pure et simple rupture avec Hegel. Contre la thèse d'une complète altérité des deux discours, l'auteur réaffirme la nécessité d'une lecture conjointe des deux corps de textes pour parvenir à une exacte intelligence de la théorie de Marx lui-même qu'elle conçoit pour sa part comme démétaphorisation de la philosophie hégélienne. C'est au jeune Marx qu'elle consacre ici son analyse, refusant de penser son rapport au Marx de la maturité en termes de dilemme et d'exclusion.

S. Mercier-Josa reconnaît avoir trouvé dans le livre de Guy Haarscher, L'Ontologie de Marx (Bruxelles, 1980), une incitation à approfondir sa propre compréhension du jeune Marx. C'est que, animé par des préoccupations apparemment analogues aux siennes, cet ouvrage débouche sur des conclusions opposées : l'idée que c'est en hégélien conséquent que le jeune Marx critique Hegel aboutit chez $G$. Haarscher à jeter le discrédit sur la pensée marxienne 
taxée d'hyperhégélianisme aux relents totalitaires. S. Mercier-Josa donne donc à sa réflexion personnelle la forme d'une discussion serrée des positions de G. Haarscher, lui opposant sa propre analyse des Manuscrits de 44 et de la Critique du droit politique hégélien pour autant que le statut de ces deux textes est la pierre de touche de l'argumentation qu'elle conteste.

S. Mercier-Josa rétorque d'abord que la critique marxienne de l'aliénation capitaliste n'est pas menée d'un point de vue éthico-juridique. La question, d'ordre philosophique, est bien plutôt celle de l'essence de la propriété privée. Comme Hegel, Marx remonte de l'en-soi de la propriété au sujet qui la pose, mais il remplace la volonté libre par le travail aliéné (p. 22). Ce dernier concept constitue donc le renversement de la déduction hégélienne de la propriété privée comme première objectivation du vouloir libre en soi et pour soi.

S. Mercier-Josa ne fait donc que tirer les conséquences de sa démonstration en affirmant que la distinction haarschérienne de l'aliénation capitaliste et de l'aliénation ontologique ne tient pas. Toute la logique des Manuscrits de 44 revient, en effet, à expliquer l'aliénation du produit par celle de l'activité, toutes les deux s'exprimant dans le rapport de l'ouvrier au non-ouvrier (p. 33). Elle suggère au passage que cette présentation du rapport social comme manifestation de la position de l'ouvrier face à son essence générique pourrait bien avoir quelque chose d'idéaliste. Nous n'hésiterons pas, pour notre part, à y voir la marque des limites de l'anthropologie feuerbachienne que Marx soumettra elle aussi à une "remise sur pieds" en 1845. Pour l'heure, le travail est saisi par Marx comme le passage à l'effectivité d'une essence humaine consistant à prendre son propre genre pour objet. Certes, il va même jusqu'à utiliser le terme d'engendrement de l'homme par lui-même, par quoi cette union de la singularité et de l'universalité est distinguée d'une donnée immédiate. Mais le processus par lequel elle se conquiert, n'en est pas moins pensé comme le déploiement d'un en-soi. L'aliénation consiste donc en ce que, d'activité générique par excellence, le travail se retourne pour l'ouvrier en perte de sa réalité humaine, séparation d'avec l'autre homme et d'avec la nature, simple moyen de la conservation de sa vie physique. S. Mercier-Josa fait alors intervenir le concept hégélien d'aliénation et en distingue, avec une remarquable rigueur, deux acceptions divergentes bien que complémentaires : la première, comme savoir de soi s'apparaissant sous la forme du savoir d'un autre; la seconde, comme dessaisissement par lequel le soi reconnaît cet autre comme lui-même. C'est cette dernière signification qui est mobilisée dans trois temps forts de la dialectique de l'aliénation de la Phénoménologie de l'esprit: Domination et Servitude, la Conscience Malheureuse, et la Culture ou l'Esprit devenu étranger à lui-même. L'auteur en compare la problématique à celle du travail aliéné dans les Manuscrits de 44 et argumente la thèse du renversement déjà formulée à propos des $\$ 44$ et suivants de la Philosophie du droit. Loin que, comme chez Hegel, l'aliénation soit le dessaisissement de soi qui élève la conscience de soi à l'universalité et la substantialité, elle décrit chez Marx la perte par l'ouvrier de sa vie générique, la manifestation de son activité propre (c'est-à-dire proprement humaine) comme une activité étrangère (p. 50-51). Comment voir dans cette critique de Hegel une radicalisation spiritualiste de sa philosophie?, conclut S. Mercier-Josa. La thèse de G. Haarscher repose en fin de compte 
sur le présupposé strinérien que toute référence à un universel transcenderait et opprimerait l'individu. Elle se fonde sur un double refus : celui de l'universel concret hégélien, et celui de l'universel concret marxien dans sa dimension spécifique (prise en charge effective de la visée réconciliatrice de Hegel moyennant précisément la dénonciation de sa stérilisation idéaliste).

A cette étape de sa réfutation, il ne reste plus à $\mathrm{S}$. Mercier-Josa, pour laver tout à fait Marx du reproche d'hyperhégélianisme, qu'à se confronter à l'interprétation haarschérienne du $\mathrm{III}^{\mathrm{e}}$ Manuscrit, c'est-à-dire à la compréhension de l'aliénation comme scission théorie/pratique. Selon Guy Haarscher, Hegel ferait preuve d'un plus grand réalisme que Marx en assignant à l'activité théorique la tâche de réconcilier, c'est-à-dire en reconnaissant le résidu d'irrationalité inhérent à la vie pratique. Ce point de vue qu'elle combat amène donc $\mathrm{S}$. MercierJosa à préciser le sens matérialiste du jugement porté par Marx sur la Phénoménologie de l'esprit et plus spécialement sur son dernier chapitre : tout d'abord, Marx crédite Hegel d'avoir saisi l'essence du travail. La dialectique de la négativité expose le mouvement général de l'aliénation qui conduit, à travers sa sursomption, à la production de l'homme générique. S. Mercier-Josa note que la reprise de cette matrice dans le III ${ }^{\mathrm{e}}$ Manuscrit permet à Marx de penser le passage de la propriété privée à la propriété véritablement humaine, dont l'articulation manquait encore aux premiers manuscrits (p. 60). L'auteur développe ensuite en les systématisant toutes les implications de la critique marxienne de la scission théorie/pratique à l'œuvre dans la Phénoménologie et reconnaît, avec Guy Haarscher, que c'est bien là le sens du concept d'aliénation dans le III Manuscrit. Mais, contre G. Haarscher, elle prouve que loin de refuser lui-même l'indépendance de la nature par rapport à la pensée, c'est sur ce point précis que Marx se démarque explicitement de l'idéalisme hégélien (p. 64). C'est parce qu'il identifie l'homme à la conscience de soi que Hegel prend pour une aliénation toute différenciation d'avec la pensée abstraite et pour la sursomption de l'aliénation le savoir de la nullité de l'objet. Marx emprunte certes à Feuerbach cette dénonciation de l'abstraction qui détache un côté de l'homme (la pensée) et l'hypostasie pour en faire le sujet du mouvement, mais il en dégage aussi les implications conservatrices, ce qui est beaucoup plus original (p. 65). $S$. Mercier-Josa estime cependant que la thèse de $G$. Haarscher contient à son insu quelque chose de vrai. La proposition hégélienne, « la conscience de soi est dans son être-autre en tant que tel près de soi-même ", quoique attaquée par Marx, habiterait son propre texte à condition d'être traduite ainsi : "L'homme devient la nature et la nature devient l'homme au terme d'un processus » (p. 72).

Sans nier pour autant l'existence d'une homologie entre ces deux énoncés, nous nous posons toutefois la question suivante : l'hypothèse d'une influence inconsciente de la proposition hégélienne sur Marx est-elle bien nécessaire? Marx ne pense-t-il pas lui-même ici comme renversement de Hegel la substitution du terme "homme" à celui de "conscience de soi »? N'est-ce pas en connaissance de cause qu'il en exhibe tous les enjeux, dont S. Mercier-Josa restitue d'ailleurs l'intime cohérence? A un oubli près cependant, la qualification marxienne du «Soi abstrait et fixé pour soi » en tant qu'égoïste abstrait, égoüsme élevé à sa pure abstraction, à la pensée. 
Tout en exprimant notre admiration pour la magistrale analyse de S. MercierJosa, nous nous risquerons à émettre ces quelques réserves:

- Ne minimise-t-elle pas sur ce point précis la différence entre Hegel et le jeune Marx?

- N'est-ce pas cette minimisation qui l'amène à écrire que Marx dans le III $I^{e}$ Manuscrit emploierait le terme d'aliénation pour nommer le rapport naturel de l'homme réel à ses objets naturels, qu'il distingue du rapport perverti de l'aliénation capitaliste (p. 70 et 71)? Or, un examen serré des textes ne nous paraît pas autoriser cette lecture.

- Nous ne souscrirons donc pas à l'idée selon laquelle le III $^{\mathbf{e}}$ Manuscrit disjoindrait de l'aliénation capitaliste le travail comme dialectique de la négativité, ce qui donnerait raison à $G$. Haarscher qui sépare le premier et le dernier sens de la notion. En effet, si l'aliénation capitaliste n'y est plus traitée pour elle-même, elle nous semble pour autant y être constamment présupposée. Notamment en relation à l'aliénation philosophique que Marx rattache, selon nous, à la première comme son effet et son symptôme. S. Mercier-Josa ne souligne pas cette liaison de la scission théorie-pratique à l'aliénation économique, qui nous parait former le point d'articulation des premiers manuscrits et du troisième. Or, n'est-ce pas parce que le travail effectif est aliéné que Hegel, tout en le présentant comme une activité générique, mystifie sa propre analyse en la déplaçant pour en faire une définition de l'Esprit?

Les nuances que nous venons d'exprimer n'entament nullement notre accord de fond avec les thèses de $\mathrm{S}$. Mercier-Josa. Nous pensons, au contraire, qu'elles les appuient dans leur visée essentielle : réfuter le paradoxe haarschérien du jeune Marx comme spiritualiste radical.

Tout aussi probante à cet égard est l'analyse qu'elle propose de la Critique $d u$ droit politique hégélien. G. Haarscher adopte lui-même la conclusion de Michel Henry - dans son livre, De Hegel à Marx (Paris, P.U.F., 1971) (Marx se proposerait ici d'homogénéiser complètement l'universel et le particulier), tout en en rejetant les prémisses (Marx refuse de mesurer le réel à l'Idée, c'est-à-dire affirme, contre la subsomption hégélienne du particulier sous l'universel, leur hétérogénéité radicale).

S. Mercier-Josa montre donc contre M. Henry que Marx ne combat pas l'Idée hégélienne comme logique spécifique de l'objet spécifique, comme unité du concept et de l'effectivité, mais seulement sa transformation en sujet, l'inversion qui fait du résultat le moteur du développement (p. 104 et 107). Bien au contraire, Marx reproche précisément à Hegel de trahir son propre principe : en faisant de l'Etat un universel a priori qui se particularise, il accueille comme vraie la première existence empirique venue, de sorte qu'en dépit de ses efforts, l'État qu'il construit échoue à être le plus haut moment de la vie sociale de l'homme (p. 93).

Ce sont donc les propres exigences de Hegel - qu'il juge pour sa part pleinement valables - que Marx retourne contre lui. L'État est chez Hegel la raison consciente de soi, mais sans s'en rendre compte, il met partout la nature en position de fondement. (C'est le cas avec la monarchie héréditaire, le majorat.) Hegel définit l'État comme l'universel concret, mais il fait en réalité de son rapport à la société civile un rapport extérieur, formel, relevant d'une 
logique de l'entendement. Quant aux instances chargées de les médiatiser, elles entérinent leur conflit au lieu de le sursumer. Est-ce à dire, comme le prétend G. Haarscher, que Marx vise à supprimer la distinction du politique et de l'économie, c'est-à-dire à en revenir à leur indifférenciation moyenâgeuse ? S. Mercier-Josa montre avec pénétration que ce que Marx reproche à Hegel, ce n'est pas d'avoir présenté l'État comme transcendant à la société civile cette transcendance est la différence spécifique de l'État moderne -, mais bien plutôt d'avoir tenté de dénier cette transcendance à l'instant même où il la reconnaissait (p. 114). Tel est, en effet, le sens de l'option hégélienne en faveur de la constitution d'états. Pourtant, Marx, pas plus que Hegel, ne se résigne au dualisme de l'État et de la société civile (p. 91). C'est ici que ce texte de Marx pose les problèmes d'interprétation les plus délicats, que S. Mercier-Josa dénoue de façon aussi subtile que rigoureuse : selon Marx, l'accès de la société civile à l'Etat ne peut se faire que par extase, transsubstantiation et non par émanation (p. 115). L'élévation à la citoyenneté du membre de la société civile ne peut se faire que par abstraction de son état social particulier.

Mais en même temps, cet État moderne abstrait n'est pas l'État par excellence. Ce qui signifie que Marx pense le suffrage universel en même temps comme le parachèvement de cette abstraction et comme sa sursomption ( $p$. 115). A cette étape de sa pensée, il charge la démocratie non d'homogénéiser le réel et le politique, comme le croient $M$. Henry et $G$. Haarscher, mais d'effectuer la conciliation de la société civile et de l'État, persuadé qu'il est, en hégélien conséquent, que la contradiction du vouloir particulier et de la visée de l'universel n'est pas insurmontable (p. 108). C'est donc au prix de la critique de l'idéalisme hégélien (l'Idée comme sujet) que Marx prend au sérieux le concept d'Esprit du peuple c'est-à-dire rétablit le peuple réel dans ses droits (p. 116).

Entièrement convaincue par la solution que $S$. Mercier-Josa apporte aux difficultés de ce texte, nous voudrions seulement, avant de conclure, inviter à réfléchir sur l'un des problèmes qu'elle soulève elle-même à la fin de son examen : quel est le rapport entre la Question juive et $c$ Manuscrit de Kreuznach rédigé quelques mois auparavant? Soulignant l'extraordinaire mûrissement intervenu entre ces deux textes, nous suggérerions pour notre part que, dans la Question juive, le critique de l'abstraction de l'Etat moderne n'épargne plus la démocratie. Disparaissent alors les contradictions qui habitent la Critique sous la forme de cette mystérieuse sursomption de l'abstraction opérée par la démocratie. Marx n'attend donc plus alors d'aucune forme d'État, fût-ce la plus achevée, la réalisation de l'universel concret. Non que ce concept, à dater de ce moment, ait perdu pour lui toute pertinence. Mais l'étude du système capitaliste et de ses modalités contradictoires d'universalisation conduira Marx, à ce qu'il nous semble, à enter sur les tendances historiques elles-mêmes la possibilité du communisme comme universel concret remplaçant l'universalité abstraite des individus dans le capitalisme. S. Mercier-Josa note elle-même dans son épilogue esquissant les transformations ultérieures de la problématique marxienne, qu'avec la rupture avec Feuerbach disparaitra l'idée de l'immanence immédiate de l'universel à l'individu singulier (p. 134), c'est-à-dire, à notre avis, le point de vue de l'universalité abstraite.

Nous ne rendrions pas complètement justice à cet ouvrage, qui unit à la rigueur 
l'audace d'une vision renouvelée de Marx, si nous ne signalions pas pour finir les fécondes perspectives tracées par le premier article donné en appendice: Est-ce "l'Esprit"? Est-ce "le Capital»? "L'Esprit", est-ce "le Capital"? Comparant ce qu'Aristote dit de l'Evepyeı $\alpha$ divine, Hegel de l'activité de l'Esprit, et Marx de celle du Capital, il établit que le Marx de la maturité ne se contente plus de dénoncer comme inversion philosophique la vision hégélienne de l'Idée comme sujet mais analyse dans le mouvement du capital son contre-type réel.

Françoise BeLLUE.

G. E. Moore et la genèse de la philosophie analytique. Prés., trad. et notes critiques de Françoise ARMENGAUD. Paris, Klincksieck, 1985. $15 \times 21,204$ p. (« Epistémologie »).

On connaît mal en France la pensée de George Edward Moore et, malgré l'étendue de leur influence sur la communauté universitaire anglo-saxonne, on n'y a guère étudié ses œuvres, restées jusqu'à présent inédites dans notre langue. Aussi faut-il se réjouir de la publication de cet excellent petit livre, qui répare, en même temps qu'un singulier oubli, une profonde injustice. Avec Russell, dont il fut l'ami à Cambridge, Moore compte parmi les principaux artisans de la «révolution analytique " intervenue au début du siècle en philosophie. La réfutation qu'il donna de l'idéalisme en 1903 passe pour avoir refermé la parenthèse spéculative ouverte quelque vingt ans plus tôt, en Grande-Bretagne, par l'émergence du courant néo-hégélien. Ses Principia Ethica (parus en 1903, eux aussi, comme les Principles of Mathematics de Russell) allaient devenir la bible des écrivains et des artistes réunis dans le fameux groupe de Bloomsbury. Au lendemain de la Seconde Guerre mondiale, Austin et Strawson ont salué en lui le précurseur d'une nouvelle forme d'analyse, fondée sur la clarification du langage ordinaire. L'cuvre de Moore, en somme, aura été une source d'inspiration et un modèle pour trois générations successives d'intellectuels britanniques. C'est assez dire son importance, et combien il est heureux que soient enfin rendues accessibles au lecteur français des pages qui font, depuis longtemps déjà, figure de «classiques " en Angleterre et aux États-Unis.

Plutôt qu'un ouvrage complet, Françoise Armengaud a préféré (dans un premier temps ?) traduire quelques-uns des articles, parmi les plus influents, d'un auteur dont ce fut le mode d'expression privilégié. Le soin qu'elle a pris d'introduire l'une après l'autre ces quelques traductions aussi élégantes qu'exactes, extraites d'une thèse de troisième cycle soutenue en 1982 à l'université de Rennes, entre pour beaucoup dans l'intérêt du livre, qui, sous l'apparence modeste d'un simple recueil de textes choisis, constitue, en ce sens, une véritable initiation à l'œuvre de Moore. De "La nature du jugement" à la "Preuve qu'il y a un monde extérieur ", chaque article donne lieu à une courte étude, destinée à en exposer les enjeux ainsi que le contexte historique de parution. Un essai liminaire d'une dizaine de pages, de nombreuses notes critiques et 
une bibliographie détaillée accompagnent également cet ensemble de textes échelonnés entre 1899 et 1939.

Les «sept articles capitaux " qu'on trouvera ici réunis correspondent aux principales étapes d'une carrière philosophique «truffee ", comme l'écrit Armengaud, " de réfutations de l'idéalisme sous des formes diverses ». Ils en résument, par-là même, le développement, à compter de la rupture de leur auteur avec le kantisme et l'hégélianisme. On ne peut qu'être frappé, en relisant ces textes, par la constance d'une démarche qu'à juste titre Armengaud qualifie de " maïeutique ", tant il est vrai qu'elle trouve son point de départ dans une sorte d'étonnement, ou de perplexité, devant la philosophie elle-même, devant les positions souvent extravagantes de ses représentants les plus éminents, devant son étrange propension à mettre en doute les croyances communes les mieux établies. Sans doute, contre l'idéalisme et le scepticisme, Moore a-t-il employé successivement les « armes d'un platonisme extrême (au moins jusqu'en 1903), puis celles du sens commun, qui, dans cette fonction polémique, prend la suite du "platonisme" " (p. 18). Mais s'il convient, de ce point de vue, de diviser en deux (et même en trois) périodes relativement distinctes son évolution, la philosophie de Moore présente malgré tout une incontestable continuité. Non que cet éternel questionneur ait jamais prétendu donner à ses idées la forme d'un quelconque système. La cohérence de sa pensée réside, non dans une doctrine, mais dans l'insistance qu'elle met à revenir sans cesse sur les mêmes problèmes - notamment sur celui des objets de la perception - pour en reprendre à nouveaux frais l'examen. Elle n'en repose pas moins sur un certain nombre de thèses, les unes « critiques », les autres « positives », dont Armengaud (qui en dénombre, pour sa part, une quinzaine) s'est efforcée de dresser l'inventaire et qui vont du principe des relations externes à l'affirmation d'une "pluralité réglée des formes du savoir " (p. 14-16). La plus importante d'entre elles - et celle qui résume, à sa manière, toutes les autres - porte sur l'indépendance de l'objet connu vis-à-vis du sujet connaissant, d'où découle, en particulier, la nécessité de distinguer entre l'objet de l'expérience et cette expérience elle-même. C'est elle qui fixe, dans son sens le plus général, le contenu du réalisme mooréen et qui confère, dans le même temps, à l'analyse sa pleine légitimité.

A la différence de Wittgenstein, auquel on l'a souvent comparé, Moore n'entend pas, il est vrai, contester aux problèmes philosophiques leur caractère d'authentiques problèmes. Loin de lui, par conséquent, l'idée de réduire la portée de l'analyse à sa seule dimension critique ou "thérapeutique ». Une même préoccupation traverse de part en part son ceuvre, touchant à la nature de la réalité, à l'objectivité de la connaissance, à la validité de l'image à la fois scientifique et commune du monde. On notera que le sens commun, pour Moore, ne s'oppose pas - ou, du moins, qu'il ne s'oppose pas fondamentalement à la science, pas plus qu'il ne s'oppose, d'ailleurs, à la philosophie en tant que telle : seules sont en infraction avec lui les errances d'une certaine forme de philosophie spéculative ou les hypothèses, non moins outrées, dont se nourrit en retour le scepticisme. Reste que, contrairement aux propositions philosophiques, les propositions du sens commun ont besoin d'être clarifiées, mais non d'être justifiées - car, si leur sens peut faire problème, leur vérité ne saurait 
être mise en cause. Dans un cas comme dans l'autre, ni l'objet ni les résultats de l'analyse ne sont, aux yeux de Moore, d'ordre essentiellement linguistique ( "laissons les questions verbales aux faiseurs de dictionnaires et autres littérateurs ", Principia Ethica, I, $\$ 2$ ). C'est donc au prix d'un certain malentendu qu'à Oxford, dans les années cinquante, on a cru voir en lui le père, et comme le premier théoricien, d'une philosophie «du langage ordinaire ». Rejoignant sur $œ$ point, comme sur beaucoup d'autres, l'interprétation de Ayer ${ }^{\mathrm{I}}$, Armengaud souligne avec raison que l'analyse des significations ne se sépare pas, chez Moore, d'un projet d'ontologie générale, que la défense du sens commun n'a jamais été, pour lui, une fin en soi et qu'enfin ce précurseur de la " montée sémantique " fut un philosophe de la connaissance plutôt qu'un philosophe du langage. Luimême, du reste, a clairement indiqué quelles étaient, dans son esprit, les deux tâches prioritaires de la philosophie: d'une part, "donner une description générale de la totalité de l'univers", en mentionnant les principales catégories d'entités que nous savons s'y trouver; déterminer, d'autre part, la nature de la connaissance elle-même (Some Main Problems of Philosophy, p. 1).

Or les deux tâches sont étroitement solidaires et la description de l'ameublement du monde doit d'abord répondre aux exigences de la logique et de l'épistémologie, comme on le voit dans le cas du réalisme propositionnel défendu par Moore en 1899 - c'est-à-dire au moment où il achève de se libérer de la tutelle de Kant et de Hegel - dans l'important essai sur la nature du jugement qui figure en tête du présent recueil. L'article est entré dans la légende du Mouvement analytique pour lui avoir servi, en quelque sorte, de manifeste et pour avoir fourni à Russell « comme par une harmonie préétablie » les concepts philosophiques dont sa logique avait besoin (J. Vuillemin). Moore y prend pour cible un passage de la Logique de Bradley consacré à la définition de la vérité, mais l'usage pour le moins ambigu du mot « idée " qu'il relève sous la plume du chef de file de l'école néo-hégélienne l'amène à dénoncer la confusion entre logique et psychologie qui sous-tend, de façon plus générale, la doctrine idéaliste du jugement et de la représentation. En ce sens, il rejoint les efforts d'un Frege ou d'un Meinong pour octroyer aux objets de la pensée - à la manière déjà de Bolzano - un statut ontologique susceptible d'assurer leur indépendance à l'égard de l'esprit humain. La vérité ne saurait dépendre d'une relation entre nos idées et la réalité, puisqu'une proposition n'est composée «ni de mots ni de pensées, mais de concepts ", et qu'à leur tour les concepts doivent être regardés comme des entités à part entière telles qu' "il est indifférent à leur nature que quelqu'un les pense ou non" (p. 50). Moore, comme le rappelle Armengaud, ne dispose pas, dans " La nature du jugement », de la distinction frégéenne entre sens et référence, ni de celle de Meinong entre subsistance et existence. D'où l'aspect quelque peu étrange et - comme le philosophe l'admet lui-même - relativement " paradoxal » d'un platonisme qui, partant du postulat selon lequel l'existence, elle aussi, est un concept, finit par supprimer toute opposition entre les concepts et les existants (p. 54). Dans les Principles of Mathematics, Russell empruntera à son jeune collègue (et ancien condisciple)

1. A.J. AYER, Russell and Moore : The Analytical Heritage, Londres, Macmillan, 1971. 
de Trinity College le double principe de la réalité des termes du jugement et de la nature non existentielle des propositions, ainsi que la thèse du pluralisme métaphysique, conséquence du principe des relations externes. Par contre, il rejettera la vision d'un monde "constitué de concepts" suggérée par Moore dans « La nature du jugement », au profit d'une ontologie bipartite fondée sur la dichotomie de l'être et de l'exister.

L'année même (1903) où, dans la Préface des Principles, Russell reconnaît publiquement sa dette envers Moore (" avant d'apprendre ces idées de lui, je me trouvais totalement incapable de construire aucune philosophie de l'arithmétique "), celui-ci fait paraître dans la revue Mind sa célèbre "Réfutation de l'idéalisme ". Cette fois, son objectif est d'isoler dans le système de l' " idéalisme moderne " un énoncé considéré comme principiel dont il se propose, au terme d'une longue analyse, d'établir la fausseté. Que peut signifier, en effet, l'affirmation selon laquelle « la réalité est spirituelle ", sinon que tout ce qui a la propriété d'être a aussi la propriété d'être objet d'expérience? Mais que signifie à son tour le fameux principe : esse est percipi, que tous les idéalistes, depuis Berkeley, semblent reprendre à leur compte? On ne saurait raisonnablement y voir l'assertion d'une relation de synonymie entre les deux termes, et d'ailleurs ce n'est pas de mots qu'il s'agit ici. Quant à l'interpréter comme une proposition synthétique a priori ( $œ$ qui la rendrait proprement inattaquable), " aucun idéaliste n'a jamais adopté cette position ». Mais s'il s'agit d'un jugement analytique, alors la thèse de Berkeley est à elle-même sa propre réfutation, tant il est clair que la perception et l'objet de la perception sont par nature deux choses distinctes.

C'est à un "sophisme " d'un tout autre ordre que s'attaquent, cette même année 1903, les Principia Ethica, dont F. Armengaud a eu la bonne idée de reproduire ici la préface ainsi que le premier chapitre, et dont il est d'ailleurs à peine croyable qu'aucune traduction intégrale ne soit, à ce jour, disponible en français. Convaincu que "bon " est une notion simple et, au même titre que "jaune", indéfinissable, que les valeurs normatives en général sont inanalysables et qu'en ce sens les jugements éthiques ne sont pas susceptibles de preuve, Moore y soutient une forme d'intuitionnisme moral dirigée aussi bien contre Bentham et Spencer que contre l'éthique « métaphysique » de Kant. Dans sa présentation, Armengaud évoque l'influence considérable exercée par les derniers chapitres du livre - par leur rejet de toute morale utilitaire et leur éloge des " plaisirs esthétiques" - sur les intellectuels cambridgiens du cercle des "Apôtres", puis sur le groupe de Bloomsbury. Avec le recul du temps, l'ouvrage de Moore retient surtout l'attention par son analyse du "sophisme naturaliste" (naturalistic fallacy), autrement dit de l'illusion qui consiste à vouloir définir les valeurs morales en termes non moraux. De même, en effet, qu'en décrivant le type de vibrations lumineuses qui doivent affecter l'œil pour que soit perçue la couleur jaune, nous ne définissons pas ce qu'est le «jaune » en tant que qualité sensible, de même « trop de philosophes » ont cru qu'en faisant l'inventaire des caractéristiques naturelles que possèdent toutes les choses bonnes ils parviendraient à définir vraiment ce qu'est le «bon " (p. 102). L'intérêt de l'argument tient au moins autant à sa structure qu'à son contenu spécifiquement éthique. Par le biais du concept de "survenance " 
(supervenience), D. Davidson, voici quelques années, en a transposé le principe dans le domaine des relations entre le physique et le mental : ainsi, le fait qu'il soit impossible que deux objets (événements, états, etc.) s'accordent dans toutes leurs caractéristiques naturelles et qu'en même temps ils diffèrent sous l'angle de leurs propriétés psychologiques ne signifie pas que les secondes soient réductibles aux premières, encore qu'il puisse exister entre elles une relation de dépendance ${ }^{2}$.

La défense du sens commun, «par quoi Moore, si modeste, acceptait cependant de se singulariser ", ne fait qu'un, selon Armengaud, avec sa profession de réalisme (p. 37). On sait qu'elle occupe, au fil des ans, une place de plus en plus importante dans son cuvre, en liaison avec le thème de la perception, de ses objets et de son rapport à la réalité physique. Mais justement, dans quelle mesure l'analyse somme toute assez complexe que propose Moore de l'expérience sensible, et surtout le recours qu'elle implique au concept de sensedatum, sont-ils compatibles avec le principe du réalisme en général, avec le réalisme prétendument " naîf " du sens commun en particulier? F. Armengaud répond en partie à cette question lorsqu'elle observe (p. 18) - de façon un peu elliptique, il est vrai - que les sense-data, en dépit de leur caractère privé, " servent » le projet réaliste (sans doute parce qu'en nous délivrant des « images sensorielles " et autres "contenus psychiques », ils restituent à la sensation son caractère authentiquement relationnel). Mais, d'autre part, il semble que la référence aux sense-data fasse partie, selon Moore, du contenu implicite de nos jugements de perception les plus courants. Aussi s'efforce-t-il, dès 1919, de concilier une théorie ouvertement représentationnelle de la perception avec l'idée selon laquelle les objets physiques sont effectivement perçus, et non (comme l'ont pensé de nombreux philosophes, y compris Russell) inférés ("Sur quelques jugements de perception ", Philosophical Studies; dans le présent volume : p. 112-134). La stratégie qu'il adopte consiste à faire dépendre la perception des objets physiques de celle des sense-data, tout en suggérant que le sense-datum associé à la perception d'un objet matériel pourrait bien, en fin de compte, être identique à la surface, ou à une partie de la surface, de cet objet. Dans l' "Apologie du sens commun " (1925), il s'emploie - par la méthode dite du "picking out " - à définir le terme de "sense-datum " de manière à laisser en suspens la question de savoir si « le sense-datum que je vois maintenant en regardant ma main et qui est un sense-datum de ma main, est ou non identique à cette partie de sa surface que je vois effectivement maintenant $»$ (p. 155). "Un peu d'humour ", estime Armengaud, est requis pour prendre plaisir à la lecture de cet article, où Moore commence par énumérer une liste de propositions dont il affirme savoir avec certitude que chacune d'entre elles est vraie, une série de truismes si " évidents ", comme il l'écrit lui-même, qu'il peut paraitre " oiseux de les énoncer» (p. 135). Mais que dire alors de la «Preuve qu'il y a un monde extérieur » (1939) - dernier article du recueil, qui comprend également une intéressante réponse à Braithwaite et à Ryle sur les objets

2. Donald Davidson, Essays on Actions and Events, Oxford, Clarendon Press, 1980, p. 253 sq. 
inexistants et le statut sémantique des noms propres en contexte de fiction ( Les objets imaginaires ", 1933) - et de la manière dont Moore, en levant simplement ses deux mains, prétend établir ipso facto l'existence d'au moins deux objets matériels? La "preuve ", si elle a fait couler beaucoup d'encre, n'a guère convaincu. C'est qu'à l'évidence, et de son propre aveu, Moore y présuppose l'essentiel, à savoir le caractère à ses yeux indubitable de la " prémisse " ("Voici une main, et en voici une autre »). Mais on peut aussi, avec Wittgenstein, lui adresser le reproche inverse: non pas d'avoir échoué à relever le défi du scepticisme (encore que tel soit manifestement le cas), mais d'avoir cru qu'il y avait un sens à y répondre. L'erreur de Moore, selon le philosophe viennois, est d'être tombé dans le piège que lui tendait son adversaire (sceptique ou idéaliste). Il n'a pas vu que la possibilité du doute ne prend effet que dans un certain jeu de langage et qu'à l'intérieur de celui-ci certaines propositions - du type de celles qu'il énumère lui-même au début de l'Apologie : "il existe un corps vivant qui est mon corps ", " la terre existe depuis de nombreuses années $॥ . . .-$ se trouvent nécessairement soustraites au doute, comme des gonds sur lesquels tourne l'ensemble de nos questions et de nos investigations. Or, "si je veux que la porte tourne, il faut que les gonds soient fixes " (De la certitude, § 343). Les arguments de Moore contre le scepticisme, et surtout les commentaires critiques qu'ils ont inspirés à Wittgenstein dans les derniers mois avant sa mort, ont incontestablement une dimension pragmatique. Mais faut-il, pour autant, n'y voir qu'une sorte d'anticipation des actuelles recherches "dialogiques" de F. Jacques (p. 38-40)?

On imagine qu'il n'était pas facile de restituer le rythme si particulier de la phrase mooréenne, allongée qu'elle est « de tant de précautions et d'incises destinées à conjurer trois ennemis : le vague, l'ambiguitté et le malentendu " (p. 43). A un ou deux anglicismes près ( "conclusif», en français, n'est pas vraiment synonyme de "concluant»), F. Armengaud s'est acquittée de cette tâche avec bonheur et sans rien perdre - rassurons-la sur ce point (ibid.) de l' « alacrité » de son modèle. Son excellente analyse introductive, si elle n'évite pas toujours certaines redites, témoigne des mêmes qualités d'écriture. Souhaitons qu'après ce choix de traductions elle nous donne à présent sur Moore l'étude de longue haleine qui fait encore cruellement défaut dans notre langue.

François Clementz.

Herman Parret, Les Passions. Essai sur la mise en discours de la subjectivité. Bruxelles, Mardaga, 1986. $15 \times 22,199 \mathrm{p}$.

M. Herman Parret s'attaque, avec les moyens de la sémiotique, à un problème très ancien et très difficile de la philosophie : celui de l'organisation des passions. Son livre comporte trois sections dont le projet d'architectonique, pris entre le chapitre des "Positions" et celui des «Exemplifications", est sans doute la pièce maîtresse. Le projet est ambitieux et sous ce concept d'architectonique qui promet " une reconstruction théorique du pathique aux différents paliers 
de sa génération et de sa manifestation " (p. 61), le lecteur ne peut oublier l'acception aristotélicienne et surtout kantienne du mot et attend une théorie de ce qu'il y a de scientifique dans notre connaissance concernant le sujet proposé, ici celui des passions. La rhapsodie des recherches et des résultats trouvés depuis la Rhétorique d'Aristote consterne encore aujourd'hui celui qui se livre à l'étude de l'affectivité et qui voit en elle un problème fondamental de la philosophie. Tenter une logique des passions semble donc au plus haut point souhaitable : le livre de $M$. Parret est écrit pour y contribuer.

Mais pour qu'un projet d'architectonique puisse emporter notre adhésion, il faut qu'il réponde à plusieurs critères qui vont nous permettre de mesurer la portée de l'ouvrage proposé. D'abord, un projet de systématisation de connaissances dans un domaine ne peut se passer d'une analyse scrupuleuse de ce domaine; sinon on n'échappe pas à l'arbitraire du dogmatisme et l'on peut se demander si la logique que l'on prétend effectuer des passions est bien une logique des passions et non une logique qui les prend pour prétexte. Il ne peut $\mathrm{y}$ avoir d'architectonique sans critique des méthodes. Ensuite, la déduction des éléments du système des diverses passions devrait être prouvée non seulement par sa cohérence interne, mais par un schématisme qui seul assure un retour à l'expérience. Enfin, on est en droit d'attendre d'une architectonique qu'elle nous offre moins une addition accidentelle de problèmes que leur articulation organique. Or, sur ces trois points, le projet de l'auteur nous laisse sur notre faim.

Sur le premier point, le fil conducteur qui permet de se repérer dans le domaine des passions est donné par la triplicité des Critiques kantiennes (p. 67). M. Parret distingue les passions chiasmiques (qui correspondent aux modalités théoriques, actualisantes et virtualisantes), les passions orgasmiques (dont les modalités sont pratiques) et les passions enthousiasmiques ou esthétiques (qu'il estime jouer un rôle équivalent par rapport aux deux types précédents à celui du jugement de goût par rapport au jugement théorique et au jugement pratique). L'idée de ce classement est intéressante mais elle reste métaphorique dans son expression tant que l'auteur ne se demande pas si les catégories de l'affectivité sont les catégories de l'activité de connaître, celles de la pratique et si le jeu réciproque de ces catégories peut rendre fondamentalement compte de l'essence d'un affect. Sur ce point, le travail de $M$. Henry est irremplaçable et fixe aujourd'hui, pour toute prétention logique dans le domaine de l'affectivité, le niveau d'exigence sans lequel un discours ne porte pas encore - et même ne porte pas du tout - sur le sentiment et usurpe le titre de « logique des passions ». Ce n'est pas parce qu'on peut opposer, dans l'analyse des passions, un sujet à un objet, distinguer l'objet de la cause, que la structure d'une passion peut être alignée sur celle de l'activité de connaissance, quand bien même on pourrait déceler une activité judicatoire à l'œuvre dans l'affectivité. La logique des passions n'est pas celle des actes de connaître, même si elle peut les croiser et s'en inspirer. De même, ce n'est pas parce qu'on peut rendre compte de certaines passions par des structures pratiques (le devoir, le pouvoir et leur combinaison) qu'une passion est une structure pratique. Il y a loin d'une logique de l'action à une logique de la passion et ce n'est pas parce qu'on réussit la première que l'on a en main tous les instruments pour réussir la seconde. Ainsi 
savoir faire la logique de la manipulation et de la séduction (p. 98-109) n'est pas encore être en mesure de dire quelque chose qui vaille sur les passions. On ne peut ignorer par principe qu'une passion est un sentir (p. 69); et si l'on se contente de remplacer $c$ sentir par une structure logique, cognitive ou pratique, on risque de le confondre avec une attitude ou un comportement.

Ne croyons pas d'ailleurs que l'analyse transcendantale de ce sentir passionnel nous écarte fatalement du projet de logique des passions; si elle compromet toute perspective structurale qui oublierait cet élément fondamental du sentir, elle n'échappe ni à la nécessité d'effectuer une sorte de déduction de la pluralité affective, ni au problème de la relation de ces passions aux circonstances concrètes dans lesquelles elles ont lieu. $\mathrm{La}$ dialectique du problème est peut-être plus profonde que ne le soupçonnait l'auteur; il fallait s'élever jusqu'à une sorte d'antithétique générale de la question pour oser se risquer à en faire la logique sans s'égarer dans le défaut d'unilatéralité et d'abstraction. La perspective structurale est trop courte tant qu'elle n'affronte pas et ne tente pas de dépasser l'antithèse qui insiste sur le sentir de la passsion. Dès lors qu'on se propose d'imiter Kant, pourquoi ne pas le faire jusque dans les moments les plus audacieux de sa philosophie?

On encourt deux autres risques à suivre trop étroitement un kantisme frileux. Le projet critique kantien, qui implique une séparation de l'a priori et de l'a posteriori, du formel et du matériel, tend à écarter par principe de ses considérations méthodologiques des procédés de réflexion qui récusent de telles distinctions. Ainsi M. Parret s'intéresse-t-il au passage des passions les unes aux autres; mais son inspection de la possibilité ou de l'impossibilité de certains passages, menée d'un point de vue a prioriste (ou essentialiste) ne lui permet pas d'envisager une solution peut-être beaucoup plus intéressante, même sur le plan logique, à coup sûr plus conforme à la philosophie de Hume : la logique du devenir des passions ne pourrait-elle être celle de la probabilité des événements complexes que sont nos passions? Or tant que l'on prend la passion pour une sorte d'essence déductible a priori, on ignore systématiquement qu'elle peut être une synthèse événementielle qui engage, à ce titre, probablement d'autres types d'événements. On retrouve chez $M$. Parret la même répugnance que chez Kant à laisser jouer à la probabilité un rôle fondamental. Du moins les modèles de développement probabiliste (qui impliqueraient sans doute une conception plus «nominale» des passions) ne sont-ils pas évoqués.

L'obédience kantienne est à l'origine d'un autre défaut. $M$. Parret aperçoit bien que le regroupement des passions autour des pôles "cognitif» et « pratique " n'atteint pas le fondement même de l'affectivité et il se tourne vers le troisième pôle du goût, censé enfermer l'essentiel de ce fondement. Il est vrai que le goût met en cause le sentiment sans qu'on puisse le réduire ni à une connaissance, ni à une action. Mais, là encore, dans son dogmatisme logique, M. Parret semble envisager comme une solution $œ$ qui, chez Kant, était un problème. La difficulté qui nous est léguée par la Critique du jugement est celle de la nécessité du jugement de goût, de la communicabilité d'un sentiment lié à une cuvre. Si cette nécessité est affirmée - à juste titre, croyons-nous - par Kant, est-elle démontrée? Le schématisme fait l'objet d'une étude particulière dans la Critique de la raison pure où Kant expose comment 
s'articulent les catégories de l'entendement et les formes de l'intuition sensible dans les fondements nécessaires de la constitution de l'objet; mais la critique du jugement de goût impliquait une extension des analyses pour montrer la nécessité de l'articulation des affects sur les formes a priori de la représentation d'un objet. Force est de constater que le problème n'est pas résolu et que le souci du schématisme n'accompagne que l'inspection de la faculté de connaître. Or une philosophie des passions qui, pour une part essentielle, se réclame du kantisme, ne devrait-elle pas approfondir le schématisme propre aux passions?

Cette dernière question nous fait rejoindre un second pôle d'inquiétude lié à la "déduction des passions ». Il apparaît d'emblée à notre auteur que le fil conducteur de cette déduction peut être livré par les catégories de modalité. Mais pourquoi le croire ? L'auteur répugne à l'analyse inductive (p. 62) : n'est-elle pas pourtant le seul moyen de nous convaincre que les catégories qui trament toute passion sont des catégories de modalité? Aristote en use-t-il autrement dans sa Rhétorique pour relever les traits caractéristiques d'une passion à partir d'une variété considérable de situations où on la trouve engagée? Le mépris de l'induction et la volonté de déduire les passions à partir des catégories de modalité, qu'elles soient cognitives ou pratiques, débouchent sur une difficulté classique du dogmatisme : la distorsion entre le repérage des prétendues passions essentiellement définies par le jeu catégoriel et celui des passions réellement vécues et désignées par ceux qui les vivent. Qui reconnaîtra l'angoisse dans son caractère essentiel à travers l'attitude de vouloir ne pas savoir un secret (p. 71)? Qui reconnaîtra dans l'obéissance le caractère prétendument essentiel de « ne pas pouvoir ne pas faire » (p. 74)? N'obéit pas seulement celui qui est contraint d'obéir! Il faut avouer que l'application mécanique des carrés d'oppositions (empruntés à la logique classique) se développe généralement à partir d'une passion décrite de façon acceptable, mais qu'elle ne permet nullement, par son jeu aveugle, de reconnaittre des passions authentiquement vécues. Si l'enthousiasme est bien analysé, l'extase, l'admiration et l'inquiétude qui font partie du même carré sémiotique sont presque méconnaissables. $\mathrm{Au}$ prix d'arguties, on finit par donner des noms aux positions produites par les "carrés sémiotiques" qui fonctionnent comme autant de fausses fenêtres logiques. Mais fait-on une bonne philosophie avec des définitions nominales?

D'ailleurs la distorsion est assez gênante pour que l'auteur se soucie de la justifier en recourant à deux arguments traditionnels : la perspective lexicale de celui qui vit ses passions et se contente de les désigner ne doit pas être docilement suivie par celui qui veut les expliquer et les organiser essentiellement. « Ce serait [même] une grave erreur de faire dépendre la déduction, de l'existence d'une terminologie des passions " (p. 69). Le discours immédiat de la conscience qui vit et dit les passions est disqualifié au nom d'une logique d'oppositions estimée plus " profonde ". La passion n'est donc pas dans sa vérité par la façon qu'elle a d'être sentie. Cette conséquence conduit au deuxième argument : une passion peut être dans sa vérité alors même qu'elle n'est pas sentie comme telle. Mais qu'est-ce qu'une passion qui n'est pas sentie? N'est-ce pas un monstre aussi étrange qu'un cercle carré ?

Or le refus de chercher les médiations entre les catégories scientifiques et les désignations communes - tout de même étonnant de la part d'un 
sémioticien - pousse l'auteur vers l'adoption d'un inconscient affectif. Le recours à l'inconscient est évidemment l'un des subterfuges par lesquels le dogmatisme cherche à se masquer. Si la logique profonde des affects ne coïncide pas avec celle de la conscience immédiate, c'est évidemment cette dernière qui est une fausse conscience; la logique profonde ne saurait être d'emblée reconnue. Le recours à l'inconscient permet d'éviter à la logique profonde le statut trop modeste de simple hypothèse. Les diverses passions seront donc comprises comme des potentialités profondes ou latentes, que le sujet ne reconnaitra pas nécessairement en lui-même, mais dont le contexte psychologique, social, économique, ... permet l'actualisation sous la forme d' " émotions ». Là encore, cette idée de "contexturation" des passions, loin d'être un schématisme authentique, n'est-elle pas plutôt la transposition sans précaution, sur le terrain de l'affectivité, de ce que la philosophie transcendantale effectue sur le terrain des sciences? Il est vrai que les catégories ne sont pas des idées dont on pourrait avoir une sorte de conscience immédiate, quoiqu'elles trament toutes les autres conceptions plus ou moins engagées dans l'expérience; il est vrai aussi que le je transcendantal, dans sa fonction de connaissance, n'a pas besoin d'être éprouvé : il exerce la fonction logique de produire les règles de construction des objets. Mais peut-on aligner le sort des passions sur celui des catégories sans qu'une analyse au moins ne justifie l'analogie, autrement dit: sans que l'on nous explique ce que c'est qu'une passion qu'on ne sentirait pas? Il semble que l'effort de $\mathbf{M}$. Parret ait été de trouver des fondements passionnels dont le caractère formel ne s'accompagne pas nécessairement d'un vécu empirique. Ainsi en va-t-il de la reconnaissance, fondement de toutes les passions communautaires (p. 83) et de la sympathie humienne qui, pour être « une condition épistémologique de toute la classe des passions intersubjectives " n'en est pas pour autant « une véritable passion » (p. 178).

Certes, il ne s'agit pas de suivre sans esprit critique la désignation ordinaire des passions ; mais déclarer que « la déduction n'est pas dépendante d'intuitions lexicologiques " (p. 69) pose trop de problèmes pour ne pas laisser soupçonner que la distorsion est l'indice d'une déduction prématurée, incapable de se justifier par un schématisme qui permettrait de montrer effectivement que les passions déduites correspondent à des impressions vécues et, sans doute aussi, à leur façon d'être communément désignées. La philosophie de l'affectivité ne gagne rien à un tel dogmatisme qui définit les passions avec le même arbitraire que s'il s'agissait d'objets mathématiques; mais nous présumons que la logique n'y gagne rien non plus. $\mathrm{Si}$, au lieu d'attendre de la logique une rectification de l'expérience, on se souciait plutôt d'expliquer l'échec des tentatives de logicisation par les " carrés sémiotiques » à rejoindre cette expérience, on se trouverait peutêtre en meilleure posture pour découvrir d'autres modalités et sans doute d'autres types de logiques. Par exemple, au nom de quel dogme les logiques binaires (de la contradiction et de la contrariété) seraient-elles seules habilitées à organiser les tableaux de passions? Que signifie qu'une passion est le contraire ou la contradictoire d'une autre? Le plaisir et la douleur - si tant est qu'ils soient les sources exclusives des passions - sont-ils en rapport d'affirmation et de négation réciproques? Russell a, de ce point de vue, peut-être ouvert une voie, lorsque, dans Signification et Vérité, il montre comment plusieurs opérations 
fondamentales de la logique sont suscitées par des situations affectives concrètes, plus ou moins complexes.

Concluons ce point en remarquant qu'il n'est pas d'architectonique qui puisse se passer de schématisme. Il n'est pas de travail logique sur un objet qui puisse se détourner des diverses approches intuitives de cet objet. S'instruit-on des choses en les brusquant?

Reste la question de l'organisation des problèmes. L'auteur, de temps à autre, commence par faire une analyse phénoménologique d'une notion avant d'en effectuer la logique. Ainsi pratique-t-il lors des études de la "manipulation", de la " séduction " et juge-t-il " compatibles et complémentaires" « l'approche phénoménologique et la reconstruction sémiotique » (p. 103). Nous nous demandons s'il n'aurait pas dû généraliser cette pratique et considérer systématiquement la phénoménologie des passions comme " prolégomène à la reconstruction sémiotique » (p. 107); ce qui lui eût évité de trancher un certain nombre de problèmes avant même de les avoir posés : une passion peut-elle être une forme transcendantale sans être elle-même sentie? D'où vient la diversité des passions ? Quel est le rôle exact joué par les circonstances dans leur apparition ?... On n'a jamais intérêt à présupposer l'essentiel : plusieurs questions fondamentales n'étant pas posées, celles qui le sont risquent de ne pas être articulées rigoureusement. Les « Exemplifications " finales, par l'impression de pêle-mêle qu'elles laissent, sont peu conformes au projet d'« architectonique ». Elles n'en contiennent pas moins, à propos d'une analyse de Spinoza, une mise en rapport importante de la réalité - en particulier, économique - avec le phénomène passionnel.

Des problèmes sont posés : y a-t-il un sens à dénombrer les passions? Doit-on distinguer entre des passions types et des passions dérivées? Y a-t-il bipolarité des passions en fonction du plaisir et de la douleur? Peut-on fonder cette bipolarité ? Comment commence et finit une passion dans une chaîne d'autres passions? Comment s'articule-t-elle avec d'autres en de multiples « parcours»? En quoi consiste la force d'une passion ou sa "tensitivite " - que l'auteur met en relation avec sa temporalité? En quoi consiste le « rééquilibrage des passions " ? Mais ils sont plus juxtaposés que coordonnés. La tâche phénoménologique, telle qu'elle a été menée récemment, n'a pourtant pas peu contribué à une telle coordination. Certes, on peut reprocher à la phénoménologie, comme le fait M. Parret, de partir du Sujet et même - le grief est plus inattendu - d'un état psychologique de croyance plutôt que des actions; on peut considérer que la passion n'est pas un phénomène exclusivement subjectif. Mais alors pourquoi l'auteur se conforme-t-il pour organiser les passions à l'architecture des trois Critiques kantiennes dont l'unité renvoie au Je pense?

C'est une chose curieuse de voir combien les philosophies des passions, même si elles ne sont pas par principe tellement tentées de suivre le kantisme, se trouvent attirées malgré elles vers une structure du type de celle de la philosophie transcendantale; comme si toutes voulaient recommencer ce rendez-vous manqué de la philosophie transcendantale et des passions.

Il faut, pour terminer, nuancer nos critiques et avouer que le livre nous a intéressé de la première à la dernière ligne. Les essais scientifiques sont assez rares en ce domaine pour qu'on doive en saluer l'effort. Sans doute échoue-t-il 
partiellement dans la réalisation de son projet, et pour des raisons profondes; mais au moins en dresse-t-il constamment l'idée. L'ouvrage ne vaut pas seulement par la qualité des analyses de détail et par ses intuitions. Peut-être est-il mû par la volonté impatiente de synthétiser de multiples et riches travaux de détail fournis autour de la question de l'affectivité par les sémioticiens auxquels il est fait référence dans les nombreuses notes de l'ouvrage. Mais toute reprise du projet d'architectonique devra tenir compte du résultat de $\mathbf{M}$. Parret, en approfondissant la déduction de telle sorte que ne s'ouvre pas un hiatus trop béant entre la tentative de logicisation et le sentir des passions. Peut-être alors sera-t-il possible de dire sur le mode philosophique et non sur celui de la simple conviction que " toute logique est affective" (p. 141) et que "la pensée même est passionnelle, le raisonnement même est affectif, et la rationalité nécessairement émotive ».

Jean-Pierre Cléro.

Objektivationen des Geistigen. Beiträge zur Kulturphilosophie in Gedenken an Walther Schmied-Kowarzik (1885-1958). Hrsg. von Wolfdietrich SCHMIEDKowARZIK. Berlin, Dietrich Reiner Verlag, 1985. 13,5 × 20,7, 426 p. ( « Schriften zur Kultursoziologie ", Bd 5).

Ce volume, qui réunit les actes d'un colloque tenu à l'université de Vienne et au Musée d'ethnologie à l'occasion du centenaire de la naissance du philosophe autrichien peu connu Walther Schmied-Kowarzik (1885-1958), disciple de Friedrich Jodl et de Wilhelm Dilthey, reprend au pluriel le titre de l'un de ses ouvrages, Die Objektivation des Geistigen (1927). Il contient deux textes du philosophe, une présentation de son œuvre avec biographie et bibliographie rédigées par son fils Wolfdietrich Schmied-Kowarzik, lui-même professeur de philosophie, et vingt-huit contributions réparties en cinq sections : Connaissance et conscience, Culture et histoire, Morale et humanite, Mythe et art, Souvenirs personnels.

Walther Schmied-Kowarzik n'est pas un philosophe de premier plan. Je laisse pour la fin la biographie du philosophe qui s'est déclaré ouvertement partisan du régime national-socialiste et s'en est repenti après la guerre. Certains des auteurs qui ont contribué à ce volume ont fui le régime nazi, par exemple Kurt R. Fischer. Quant à l'œuvre de Schmied-Kowarzik, elle mérite d'être tirée de l'oubli et mise en perspective historique qui indique l'aboutissement de ses lignes de recherche. C'est ce qu'ont réussi les auteurs réunis dans cet ouvrage, en mettant en évidence les contributions essentielles de Schmied-Kowarzik à l'évolution des problèmes philosophiques, qu'il s'agisse du renouvellement du problème de l'espace, de la constitution de la théorie de la connaissance à partir d'une psychologie non empirique ou de l'interprétation des mythes.

Kurt R. Fischer esquisse l'arrière-plan philosophique viennois au début du $\mathrm{XX}^{\mathrm{e}}$ siècle dans lequel s'inscrit la pensée de Walther Schmied-Kowarzik. C'est d'abord Brentano et son école (Meinong, Ehrenfels, Twardowski auxquels il 
faudrait ajouter Masaryk) : l'enseignement de Brentano constitue un point de départ non seulement pour la phénoménologie et pour Heidegger, mais aussi pour Freud qui a suivi ses cours pendant quatre semestres. Après Brentano, c'est Mach qui deviendra la figure dominante de la philosophie autrichienne, si bien que le Cercle de Vienne se réclamera de son héritage autant que du Tractatus de Wittgenstein. Kurt Fischer a raison de rappeler que tous les grands courants de la philosophie du $\mathrm{Xx}^{\mathfrak{e}}$ siècle se rattachent, d'une manière ou d'autre, à la philosophie autrichienne : la phénoménologie, la psychanalyse, la philosophie de Heidegger, l'empirisme logique, la philosophie analytique.

La brève mais substantielle contribution de Richard Heinrich, l'une des plus importantes de l'ouvrage, analyse les théories philosophiques de l'espace dans le premier tiers du $\mathrm{xx}^{\mathrm{e}}$ siècle, y compris celle de Walther Schmied-Kowarzik. Selon R. Heinrich, le concept d'espace a subi une transformation profonde au cours du $\mathrm{XIX}^{e}$ siècle : l'espace n'est plus déterminé par sa structure géométrique. Or, l'espace, concept éminemment philosophique aux XVIr et $\mathrm{XVIII}^{e}$ siècles, fournissait la clé de la compréhension de la physique. Ce concept appartient désormais aux physiciens (comme l'avait d'ailleurs déjà rappelé Otto Neurath), et sa structure sera ou bien déterminée empiriquement, ou bien posée par convention. La philosophie a donc perdu le concept d'espace. Ce concept a éclaté : on peut tout juste parler de théories anthropologiques, mathématiques, esthétiques, peut-être même philosophiques de l'espace, mais ces différentes conceptions ne déterminent pas la forme de la théorie elle-même comme $œ$ fut le cas par exemple pour Descartes. La philosophie se trouve donc dans une situation dans laquelle «quelque chose qui a été compris jusque-là ne sera peut-être plus compris - au sens philosophique " (p. 70). Il faudra reprendre l'une des dernières tentatives pour construire une théorie unitaire de l'espace à partir des concepts d'espace formel, espace intuitif et espace physique, qui est celle de Carnap (Der Raum. Ein Beitrag zur Wissenschaftslehre, 1921-1922) et qui s'inspire aussi bien de Russell que de Husserl.

En revenant à des conceptions mentalistes et psychologisantes (les concepts sont des représentations mentales), Erhard Oeser emprunte un ton qui tranche avec la philosophie analytique dans sa version linguistique. Confrontant la philosophie kantienne du cerveau avec les tendances actuelles de la neurobiologie, il met en relief la conception fonctionnelle de l'esprit chez Kant dans les controverses du XVIII ${ }^{e}$ siècle sur le siège de l'âme (S.T. Soemmering).

Parmi les autres contributions philosophiques, celle de Michael Benedikt traite de Kant et de l'éthique matérielle des valeurs au $x x^{e}$ siècle; celle d'Erik Heintel ( « Le point comme problème philosophique fondamental ») analyse les concepts leibniziens de point géométrique, point physique, point métaphysique (monade) et leurs prolongements chez Kant et chez Hegel. Le lecteur français pense au chapitre "Le point fixe» dans Le Systeme de Leibniz de Michel Serres, écrit vingt ans plus tôt et plus pertinent. A partir des travaux sur le sommeil et le rêve, Walter Pöldinger compare les rôles de l'hémisphère droit et de l'hémisphère gauche du cerveau, et rattache les questions de psychologie profonde à la mythologie.

Les articles consacrés au pluralisme culturel, à la philosophie de l'histoire et à la philosophie des valeurs ne sont pas moins importants. Si Paul Feyerabend 
préconise le pluralisme et l'opportunisme culturels contre la conception autoritaire de la rationalité qui s'abrite derrière les arguments et les démonstrations ( « ce sont des concepts vides qui mènent aux démonstrations strictes - et plus ils sont vides, plus les démonstrations sont strictes ", p. 141), dans un article nuancé, Justin Stagl se prononce pour un relativisme modéré. A son avis, le relativisme culturel radical, par exemple celui de Sartre dans la Préface aux Damnés de la terre de Frantz Fanon, "n'est en aucune façon plus aimable que la foi européocentrique dans le progrès, par laquelle on a justifié aussi bien le système colonial que l'exportation des idéologies révolutionnaires ou les établissements locaux de bonheur forcé " que l'auteur appelle de façon si expressive en allemand lokale Zwangsbeglückungsanstalten (p. 154). La philosophie structuraliste de l'histoire dont un des représentants est Hyden White (Metahistory. The Historical Imagination in Nineteenth-Century Europe, 1973) a trouvé son critique en la personne de Herta Nagl-Docekal. Peut-on ramener l'explication en histoire à un acte métahistorique, poétique, selon les tropes de métaphore, métonymie, synecdoque et ironie? Un résumé aussi brutalement schématique de la thèse de White suggère une réponse négative, mais Herta Nagl-Docekal montre que la méthode de White pose des problèmes essentiels même si elle ne réussit pas à les résoudre. - Comment changent les valeurs dans notre société? La réponse de Peter Heintel est le développement de la thèse selon laquelle l'humanité est parvenue aux limites au-delà desquelles elle ne peut ni avancer ni reculer: limites de l'espace, celles du temps dues à l'accélération de la production, celles de l'exploitation de la nature (l'homme aurait libéré des forces qu'il ne serait plus en mesure de contrôler) et de l'organisation du travail. La tâche actuelle de l'humanité consisterait alors dans la gestion de ces situations limites. - L'humanité serait-elle parvenue aux limites de l'espace? L'espace cosmique n'offre-t-il pas un champ illimité d'expansion et un exutoire à la volonté de conquête? Un changement dans les valeurs et les attitudes ne peut-il ouvrir de nouveaux domaines, aujourd'hui insoupçonnés?

Il est impossible de parler de ce recueil d'articles consacré à l'œuvre philosophique de Walther Schmied-Kowarzik sans dire quelques mots sur sa vie. Il fut nationaliste pan-allemand (bien que le nom double indique l'origine tchèque de la famille), attentif cependant aux autres cultures. De famille catholique, il devint un "protestant libre " selon sa propre expression. Pendant six ans, il fut professeur de philosophie à l'université estonienne de Dorpat (Tartu) et épousa en secondes noces l'écrivain Gertrud von den Brincken, d'origine balto-allemande. En 1927, il fut appelé à l'Académie pédagogique de Francfort-sur-le-Main où il enseigna pendant six ans en attendant un poste à l'université. C'est à partir de l'arrivée au pouvoir de Hitler que se noua son destin.

En 1933, Schmied-Kowarzik devient Dozent à l'université de Giessen. Peu après, en juillet et août de la même année, commencent les premières purges dans les universités allemandes; ses amis August Messer et Ernst von Aster sont destitués avec une pension très réduite. En octobre 1933, ce sera le tour de Schmied-Kowarzik qui pourra néanmoins continuer à donner des cours à titre de Privatdozent, sans traitement. Poussé par ses difficultés matérielles, il se décide à rejoindre le parti nazi ; il sera alors nommé à l'École normale de 
Friedberg. Un incident éclate bientôt : lors d'un cours, il parle de Freud et d'Adler et il est chahuté par les étudiants national-socialistes qui le dénoncent. Il est renvoyé de l'École normale et c'en est fini avec l'espoir d'une carrière universitaire : il ne pourra jamais devenir professeur. Découragé, désespéré, il s'inscrit dans une école du parti, mais en même temps, dans un texte censuré, il défend les droits des minorités et des communautés religieuses, sous-entendu juives. Après des mois de grande misère matérielle et morale, il se soumet de nouveau aux examens en histoire et géographie pour pouvoir reprendre son activité d'enseignement au moins au niveau du lycée. Avec condescendance, ses anciens collègues qui font carrière sous l'égide du régime nazi - ainsi le hégélien Hermann Glockner - le dispensent des examens de philosophie. Il est un de ces personnages incapables d'envisager leur existence autrement qu'en faisant des cours devant un public qui lui est assuré d'office. D'autres ont émigré, se sont reconvertis, ont exercé mille métiers ; Schmied-Kowarzik, deux fois congédié par les nazis, ne sait mieux faire sinon devenir propagandiste du régime dans les écoles du parti. Il pourra reprendre ses cours à l'université de Giessen (sans être payé) et à partir de 1940 à l'université de Vienne, malgré l'opposition d'Arnold Gehlen, maître de la philosophie viennoise sous les nazis. Cependant, ses écrits (ainsi que les poèmes et un roman de sa femme) sont retenus par la censure; entre 1934 et 1945 , il ne peut rien publier. Sa femme refuse toute collusion avec le régime.

Dans sa petite ville, il tente d'empêcher le pillage et les destructions des biens juifs au cours de la «nuit de cristal » en novembre 1938. Le lendemain, il veut quitter le parti, mais se laisse trop facilement convaincre d'y rester afin que le parti ne soit livré aux «mauvais éléments". Il se réjouit de l'Anschluss et rentre en Autriche, désormais province du Reich allemand. C'est seulement en 1944 qu'il reconnaît le génocide des juifs et se rend compte de sa complicité avec le régime nazi et de sa propre culpabilité. Après la fin de la guerre, il se livre aux autorités alliées en tant qu'ancien membre du parti nazi, est interné et fait l'objet d'une longue procédure, alors que ceux qui avaient occupé des postes de responsabilité sont rapidement réinstallés dans leurs fonctions et dignités universitaires.

Aujourd'hui, alors que l'Autriche hésite toujours à exorciser les démons de son passé et que l'on scrute le passé nazi de Heidegger, le temps est peut-être venu de s'interroger sur des égarements analogues auxquels ont cédé d'autres philosophes, le régime nazi n'étant pas le seul en cause.

Jan Sebestik.

Francisco López Frías, Ética y politica. En torno al pensamiento de J. Ortega y Gasset. Segunda edición. Prólogo de Julián MARIAS. Barcelone, Promociones Publicaciones Universitarias, 1985. $14 \times$ 20,5, 375 p. (« Biblioteca universitaria de filosofia ", 4).

Cet ouvrage est assurément le meilleur qui ait été composé, jusqu'à présent, 
sur Ortega philosophe politique et l'on doute qu'il puisse être, à l'avenir, dépassé. Le docteur Fr. López Frías, depuis 1970 professeur de philosophie à l'université de Barcelone (après avoir enseigné aux États-Unis), est bien connu pour ses travaux sur la déontologie professionnelle, sur l'éthique appliquée et sur Ortega (cf. en 1972, La Condición del ejercicio de la Razón Vital). Comme le dit J. Marías (« Prologue », IX), " non seulement il a senti l'attraction d'Ortega, mais encore il lui a consenti". Marqué au coin d'une vaste et sûre érudition, mais plein de vie et de conviction, ce livre a connu en trois mois un tel succès que sa deuxième édition a paru la même année (avec l'ajout d'un « Epilogo sin final ").

Deux traits le caractérisent: d'une part, la réflexion scrupuleuse sur la philosophie d'Ortega, appliquée à la politique et, d'autre part, l'étude minutieuse et impartiale de la réalité espagnole de 1900 à 1936 , que veut transformer le fondateur du ratiovitalisme. "Ortega entend la politique dans sa plus intime radicalité, c'est-à-dire comme une éthique » (« Presentación », XIII). Les sept chapitres s'intitulent : «Les écrits politiques d'Ortega »; « Du XIX siècle au $\mathrm{XX}^{e}$ siècle "; "Ethique et politique "; "Les nouvelles exigences du savoir philosophique "; " La falsification de la réalité "; "Ethique et neutralité : la Première Guerre mondiale »; "La nécessité du changement ». Une large bibliographie clôture le volume. Au fil de ces pages fascinantes, on aperçoit l'unité profonde de la pensée d'Ortega, qui n'a jamais varié pour l'essentiel, dès avant sa grande conférence de 1914 ("Vieja y nueva política ») et la fondation de sa «Liga de Educación Política Española »; il a toujours vivement critiqué la stagnation de la politique de la "Restauración" (sous Alphonse XII et Alphonse XIII, avec Canovas Castillo et ses successeurs) et il s'est efforcé de promouvoir un renouvellement profond de la politique espagnole; devenu hostile à la monarchie, et fondateur de la République de 1931, il n'a pas tardé à critiquer cette dernière pour ses déviations; indépendant, il a été attaqué par les deux camps (extrême droite et extrême gauche). Parmi tant d'apports intéressants, on notera : que « la politique est, pour Ortega, la partie la plus visible et manifeste de la vie sociale " (p. 15); que sa collaboration aux journaux était pour lui une manière d'enseigner; qu'il fut socialiste de 1908 à 1914; que selon lui, « il y a en Europe des élites qui gouvernent, tandis qu'en Espagne il y a des élites qui commandent " (p. 23); que la politique dépend autant des circonstances que des grands principes qui doivent l'inspirer; que chaque région espagnole doit être autonome, sans aller jusqu'au fédéralisme; que l'homme-masse est surtout le fasciste (p. 145); que le syndicalisme est plus important encore que le parlementarisme; que la neutralité espagnole en 1914-1918 fut un leurre (Ortega signa le manifeste en faveur des Franco-Anglais en juillet 1915); que l'Espagne officielle ne se confond jamais avec l'Espagne réelle et vivante, etc. L'antifranquisme d'Ortega apparaît indéniable; mais ses dissentiments sur certaines mesures de la République ne le sont pas moins. En résumé, un ouvrage fondamental, tant pour son objectivité que pour son brio.

Alain GuY. 
Lloyd DE MAUSE, Les Fondations de la psychohistoire. Trad. de l'américain Sean Wilder, Jean-Maurice BIZIËre. Paris, P.U.F., 1986. $15 \times 22,296$ p. (" Perspectives critiques »).

Le livre de Lloyd de Mause, Les Fondations de la psychohistoire, aborde non sans héroïsme une question complexe : comment des facteurs inconscients peuvent-ils être à l'œuvre dans les événements historiques ? Comment les actions historiques des groupes et des individus peuvent-ils être l'effet de fantasmes? Répondre à ces questions exige l'élaboration d'une méthodologie fine que les articles ici présentés tentent d'élaborer avec une précision croissante. Les premiers articles restent sommaires, comme l'auteur le reconnaît lui-même. Par exemple, dans "L'évolution de l'enfance ", il construit un schéma progressif de l'histoire des sociétés qui fait de cette dernière, de l'Antiquité à nos jours, le reflet de la capacité croissante des processus psychiques inconscients à devenir conscients et, par-là, à suspendre leur réalisation immédiate. On partirait ainsi des sociétés qui tuent les enfants pour arriver, à l'époque contemporaine, à des sociétés qui aménagent de façon moins meurtrière la violence des générations antérieures face à l'enfance. Dans cette perspective, les modèles éducatifs sont proprement la matrice des processus historiques.

Lloyd de Mause ne procède donc pas comme Freud, dont il se réclame, pourtant, c'est-à-dire par des analyses qui se disent et se veulent partielles, même si elles ont de multiples conséquences sur l'intelligence d'autres aspects de la réalité historique. Non, mettant en lumière des séquences causales inédites, il en fait des causes plus fondamentales que celles que reconnaissent les historiens. Aussi ses analyses ont-elles toujours une portée polémique explicite : les modèles explicatifs qui produisent des sociétés diverses selon un mécanisme de type évolutionniste - sélection, par l'éducation, de petites différences et isolement géographique de la mutation psychogène considérée - sont vraiment la matrice des phénomènes historiques considérés. Et l'auteur ne craint pas d'opposer massivement sa méthode à celles de Philippe Ariès ou de Marx.

On choisira, de cette ambition causale de la psychohistoire, un autre exemple : repérant, dans les textes contemporains de plusieurs déclarations de guerre, des fantasmes de naissance difficiles à dissocier d'angoisses d'étranglement, Lloyd de Mause fait alors de ces fantasmes la cause du déclenchement de ces guerres (p. 160). Mais, dans le long texte "Des fantasmes de groupe historique ", il revient sur cette première affirmation : « La question n'est pas tant de savoir, en effet, si l'imagerie de la naissance est présente ou non dans le matériel historique [...] il s'agit plutôt de la signification qu'elle peut avoir et de la fonction qu'elle occupe dans la dynamique du groupe. $\mathrm{Si}$, par exemple, l'on commence par le stade "effondrement" du fantasme de groupe, la raison pour laquelle il y a toujours une prolifération violente de l'imagerie de la naissance - tout comme il y a une résurgence d'imagerie violente de caractère oral, anal et œdipien - c'est parce que le fantasme de groupe qui, auparavant, avait contenu, entravé, et constitué un rempart défensif contre le refoulé, s'est effondré. " Comprendre l'incidence de l'inconscient sur les phénomènes historiques, c'est, essentiellement, 
comprendre certaines violences à l'œuvre dans les sociétés : meurtres ou désirs de meurtres des enfants, amours archaïques indistinctes de haines massives et déplacées sur les chefs politiques, les fantasmes de groupe historiques sont, selon Lloyd de Mause, le déplacement sur la scène publique, d'affects liés à la recherche d'amour des individus.

Si cette manière nouvelle d'écrire l'histoire attaque les modèles explicatifs reçus, c'est au fond que ces modèles participent des défenses par lesquelles ces désirs sont méconnus, les historiens méconnaissant, par leurs explications rationalisantes, la violence des déterminations de groupe. Telle est la raison de l'intrication, dans ce livre, entre les analyses précises et la polémique. Mais lorsque la méthode d'analyse des fantasmes de groupe historique se précise, la polémique violente devient moins importante, et plus importante au contraire la nécessité de situer cette incidence de l'inconscient dans l'histoire des sociétés par rapport à d'autres facteurs dont la réalité n'est plus niée d'un trait de plume (p. 168).

Monique DAvid-MENARD.

Michel Henry, La Barbarie. Paris, Grasset, 1987. $13 \times 21,252$ p.

L'originalité de l'œuvre de $M$. Henry tient sans doute au rôle majeur qu'elle accorde à l'affectivité et que l'histoire de la philosophie des passions ne lui avait jusqu'alors jamais assigné : le sentiment, loin d'être un épiphénomène qui accompagne la vie pratique et l'activité théorique, est en fait le fondement de tous nos actes. Ce renversement de perspective, qui n'a peut-être d'égal que la "révolution copernicienne ", a permis au philosophe d'envisager une philosophie de la réalité et de réfléchir dans des conditions neuves à l'économie. $L a$ Barbarie, en dépit de sa taille modeste, veut élargir ces ambitions à une critique plus générale des valeurs de notre temps - le rôle de la science, celui des médias et de l'Université. La philosophie de l'affectivité ne peut-elle produire qu'un discours ironique et désespéré sur le compte de la réalité sociale ou bien est-elle en mesure de prendre date et de nous faire remarquer l'événement décisif d'une entrée dans la barbarie qui passerait inaperçu sans elle?

Dans une satire impitoyable, l'auteur dénonce l'injustice des sociétés modernes, c'est-à-dire la confusion générale des compétences, depuis la démission de ceux qui doivent transmettre la vie essentielle, avec les usurpations qui en résultent, jusqu'au règne sans partage de la télévision, promotion de l'insignifiance. Il diagnostique partout les effets de l'idéologie galiléenne qu'il caractérise par la mise entre parenthèses systématique des qualités sensibles - discréditées comme inessentielles et ne livrant pas la réalité des choses - et par la constitution (à l'aide des mathématiques) d'un monde d'objets, le sujet ayant réuni (sans bien s'en rendre compte) dans cette tâche toutes les conditions de son autodestruction. Le monde objectif que produit la science en ses représentations est devenu, par les moyens d'une technique qui n'entretient plus avec le corps 
dont elle est pourtant principiellement issue que des rapports très allusifs, notre milieu de vie et comme la raison de toutes nos valeurs. La pratique même du sujet est devenue objective (p. 85), le savoir qui règle l'action étant celui de la science (p. 89). Or la vie ne consiste pas dans l'objectivité; la réalité de la vie et des choses est précisément dans les qualités dites secondes, dans le sentir de soi, le se sentir et l'être-senti. Il n'y a de culture que dans l'autotransformation de ce sentir par la création d'œuvres ou par leur contemplation. Le monde dans lequel nous sommes désormais contraints de vivre nie la culture et s'attaque à elle. La vie produit des conditions qui l'empêchent de se développer, la reléguant au mieux à la clandestinité de la création (p. 241), la vouant le plus souvent à l'ennui (p. 191), au désespoir, à la violence car une énergie qui ne se développe pas n'a pas d'autre exutoire (p. 183). C'est cette contradiction que $M$. Henry explore sous le nom de "barbarie ". C'est sa portée qu'il nous faut apprécier, car l'auteur met en jeu dans cette satire (qui prend quelques allures d'un ouvrage pour le grand public) plusieurs principes fondamentaux de sa philosophie.

Si la tâche phénoménologique a permis de découvrir la réalité comme affective, force est de constater que la réalité, conformément aux vues du courant galiléen, paraît se confondre aujourd'hui avec l'objectivité. La philosophie de M. Henry jaillit donc dans un monde qui semble partout fondamentalement en prendre le contrepied. Dans ces conditions, peut-elle encore se prévaloir d'être une philosophie de la réalité? N'est-elle pas plutôt une revendication pathétique de la subjectivité malheureuse qui, dans sa souffrance infinie et en proie au "délire de la présomption ", voue à la malédiction et à la barbarie un monde qu'elle ne reconnaît plus être celui du développement du se sentir? N'est-elle pas vouée au sort d'observatrice redresseuse de torts s'enfermant dans le regret d'un passé indéterminé? Cette philosophie de l'affectivité est-elle une pensée qui signifie et explique la "crise" de notre temps ou n'en est-elle qu'un symptôme?

Une réflexion rigoureuse menée à partir de l'affectivité permet de déceler toutes sortes de décalages entre la réalité et l'objectivité, en dépit des efforts de notre époque pour les rendre identiques et de la croyance, universellement répandue, qu'elles le sont. L'affectivité est à la fois fragile et indestructible, car c'est elle qui produit jusqu'à ce qui la nie intimement. D'une façon très schopenhauerienne, M. Henry explique comment la vie, dans sa souffrance d'ellemême, finit par vouloir se fuir et se détruire (p. 178). Mais cette fois, ce n'est pas la nature du vouloir-vivre qui se nie pour produire la culture; c'est la culture, assimilée à la vie, qui produit la barbarie, "une nouvelle nature sans l'homme ". La production de l'objectivité comme réalité est l'invention la plus radicale de la vie pour tenter de se fuir elle-même : cet événement catastrophique serait celui de notre temps (p. 114). Ainsi la science "galiléenne" se trouve-t-elle paradoxalement rejetée hors du monde de la culture, compromettant toutes les formes qu'elle a su se soumettre. Et l'on ne doit compter sur aucune force salvatrice issue de la contradiction : la contradiction est dangereuse, peut-être terminale pour la culture. Mais cette thèse métaphysique est-elle convaincante ?

Certes l'idée d'une science se développant sui generis (p. 95) et semblant 
exclure tout enracinement dans le jugement des individus a été soutenue contre la phénoménologie husserlienne d'ailleurs - par Cavaillès, par exemple; mais pourquoi désormais prendre comme un dogme une théorie de la science qui, après tout, n'est peut-être qu'un fantasme conceptuel? Une idéologie n'estelle pas souvent l'image inversée de la réalité des choses? N'y a-t-il pas dans la philosophie de $M$. Henry qui sait guetter en chaque acte - fût-il conceptuel - son fondement affectif de quoi résister avantageusement à cette conception de la science? Il faudrait toutefois, pour continuer la résistance, adopter une autre attitude qui prendrait le temps d'analyser patiemment des gestes scientifiques singuliers, réellement effectués, pour les envisager sous l'angle de leur économie passionnelle et ne pas se contenter d'allusions vagues aux « molécules » de la biologie ou aux statistiques. D'autant que «la » science ne peut être objective en un seul sens qui serait celui d'une haine de la subjectivité et de la vie : il existe par exemple tout un secteur des statistiques, depuis leur origine bayesienne, dans lequel il s'agit pour le calculateur d'assigner des limites à l'évaluation d'une situation faite par le sujet. En vain voudrait-on couper les sciences des préoccupations vitales et sensibles des hommes; quand ces soucis ne pénètrent pas directement l'activité scientifique, toute une dialectique complexe se ramifie en tout sens pour accorder les intérêts apparemment divergents que cette activité suscite, pour réfléchir aux questions qu'elle pose, au sens de ses réponses, pour interroger incessamment la « rigueur» de ses pratiques, pour s'approprier ses résultats (comme M. Henry le fait avec l'œuvre de Freud ou celle de Janet), enfin pour retracer l'histoire des problèmes rencontrés. La science, dans sa conception «galiléenne ", peut bien repousser hors d'elle la dialectique où la philosophie a un rôle original et toujours singulier à jouer : elle ne peut pas l'éliminer; et surtout, elle ne peut ni ne songe à nous faire vivre dans le monde d'artefacts qu'elle est obligée de construire pour mettre à l'épreuve ses hypothèses. Ainsi avouons-nous avoir été peu convaincu par les craintes développées trop généralement par l'auteur à l'encontre de l'idéologie "galiléenne" - d'ailleurs elle-même trop simplifiée. Lorsque M. Henry s'efforce de déterminer par concepts sa philosophie de l'affectivité, fait les distinctions nécessaires, ne nie-t-il pas à sa façon la vie? Ne cherche-t-il pas, à son tour, à l'objectiver sur un certain mode ? Quand bien même l'affectivité se porterait, par un mouvement propre, aux distinctions nécessaires, la philosophie ne requerrait-elle pas les concepts pour les exposer? La dynamique et l'énergétique que l'auteur nous propose de l'affectivité (p. 181-182) ne s'inscrivent-elles pas, même sur le mode métaphorique, par leurs considérations quantitatives, dans un sillage plus " galiléen » que "subjectiviste "?

Si l'abstraction de la thèse soutenue sur l'activité scientifique ne semble pas résulter fatalement du projet de penser la réalité et les valeurs à partir de l'affectivité, il faut toutefois nous demander si elle ne dépend pas de quelque faille qui existerait, non seulement dans la liaison des conséquences aux principes, mais encore au niveau le plus fondamental de la réflexion de l'auteur sur l'affectivité. La philosophie de $M$. Henry n'occupe-t-elle pas l'un des versants d'une dialectique dont elle n'est pas l'antithétique? En d'autres termes, les problèmes de l'affectivité ne sont peut-être pas encore, dans leur principe, sortis 
du conflit - s'il est possible qu'ils le soient dans le cadre d'une philosophie transcendantale. Deux remarques suffiront ici pour nous en convaincre.

Si M. Henry a porté à la plus haute clarté, contre presque toutes les philosophies jusqu'à ce jour, qu'aucune structure n'épuisait l'être d'un sentiment s'il a su affirmer l'auto-affection de la subjectivité transcendantale, a-t-il pu se sortir du problème de la déduction transcendantale des sentiments, inséparable ment formes et contenus? En d'autres termes, quel est le rapport entre l'affectivite et les affects particuliers? La considération de la temporalité engagerait à remarquer de même que si les sentiments ne sont pas rythmés par la succession des situations, s'ils trouvent leur temporalité a priori dans l'historial de l'Absolu, il n'en demeure pas moins que le problème de la jonction de ces changements purs aux changements empiriques se pose. Et il n'est pas anodin pour constituer une philosophie de la réalité : si l'affectivité prend sa source hors du monde (p. 66-67), il faut tout de même reconnaître que c'est en rapport avec des situations que les sentiments sont éprouvés; dès lors un difficile problème de schématisme se pose si l'on veut éviter que la philosophie de l'affectivité ne verse dans l'effusion au lieu de penser le principe même de la réalité. N'est-ce pas le manque cruel de schématisme qui porte à soutenir des thèses métaphysiques trop générales et fait rater la dimension "dialectique " de la science? Les répercussions politiques en sont également considérables : La Barbarie dénonce et ne fait qu'en appeler, pour toute politique, au salut du monde « par quelquesuns ". Là encore, l'activité de médiation, si capitale en ce domaine, fait défaut; il y a plus : n'est-elle pas implicitement attaquée, sous quelques-uns de ses aspects, derrière les charges contre la télévision (est-elle fatalement mauvaise?), contre les journalistes (n'y en aurait-il que d'incompétents?), contre la liberté de la presse même (sous l'étrange prétexte qu'on en use mal)? Enfin, comment à la fois soutenir l'indépendance de l'affectivité à l'égard du monde et veiller à ce que l'organisation de ce monde ne blesse pas et ne dégrade pas nos affects?

Cette négligence, voire ce refus, des médiations accompagnent le mouvement de porter l'affectivité à l'absolu. Ce qui s'effectue selon deux modes, le mode " énergétique " et le mode "cognitif", dont la conciliation semble nous faire entrer dans un labyrinthe. Si l'essentiel de l'affect réside dans la «tonalité ", alors il est difficile de lui assigner un pouvoir de connaître quelconque ; il réside plutôt dans le sentir d'une force. De multiples textes développent cet aspect " énergétique " de l'affect (p. 175, p. 192). Mais l'énergétique est-elle susceptible du même traitement philosophique que la subjectivité qui est identique au «se sentir » ? Un sentiment n'est pas un fait mental - même si $M$. Henry se laisse glisser parfois vers une sorte d'innéisme affectif (p. 121); il est plutôt de l'ordre de la norme, de la règle, du principe. Comme il est une appréciation de notre être, il est difficile de ne pas le traiter comme un savoir - ce qui lui arrive chez M. Henry. Certes il ne s'agit pas d'un savoir " galiléen " par lequel un sujet s'opposerait à un objet : ce rapport de transcendance laisse s'évanouir le sentiment. On peut donc concéder à l'auteur qu'il s'agit d'un autre type de savoir. Mais si M. Henry parle de transparence du sentiment (p. 68), s'il parle de sa vérité (p. 69, 123-124), il ne parle jamais de sa possibilité d'erreur ; or un savoir qui serait toujours assuré de sa vérité resterait-il encore un savoir? 
Si nous voulons bien admettre qu'il y ait une « justesse » de la saisie affective, c'est qu'il y a aussi une possibilité de se tromper. Mais quelle est cette étrange règle qui nous permet, dans le cas de l'affectivité, de saisir le vrai et le faux?

Ces problèmes dialectiques sont beaucoup plus importants qu'il n'y paraît pour connaître la nature de la culture et celle de la réalité : d'une part, la culture est-elle le développement de soi de la vie (auquel cas la barbarie serait une sorte de maladie) ou est-elle une saisie adéquate de ce qui est vrai, juste, ... (auquel cas la barbarie serait une sorte d'erreur)? Le jeu de la puissance et de l'adéquation mériterait des éclaircissements. D'autre part, si la réalité doit être enracinée dans l'affectivité, et si l'auteur nous rappelle son caractère non substantiel et temporel (p. 109), il faut, pour la comprendre comme principe, connaître la nature des règles qui régissent l'affectivité. Ce problème, qui nous fait rejoindre par un autre tour celui du schématisme, n'est pas porté à sa plus extrême clarté.

Toutefois, il faut saluer comme un mérite considérable d'avoir apporté tant d'éléments pour une antithétique des positions sur l'affectivité. Mais guerroyer dans un écheveau d'antinomies n'est pas encore les résoudre. Une philosophie de l'affectivité qui se veut une philosophie de la réalité sous toutes ses formes doit résoudre cette dialectique. La fécondité des résultats déjà obtenus en ce qui concerne la pensée de l'économie, celle de l'art, celle du sacré, doit encourager une enquête plus délicate, mais indispensable sur le terrain des sciences. C'est en défendant la philosophie de l'auteur contre lui-même que nous contestons sa conception de la science et de la technique. Il serait donc sage de penser par provision - même si l'on place quelque espérance dans la philosophie transcendantale des passions - que les contradictions dénoncées comme étant celles de notre société sont partiellement obscurcies par celles mêmes que doit encore affronter la philosophie des passions qui aura connu sa dialectique plus tardivement que la raison issue de la science galiléenne. Par un étrange retournement des choses, l'exigence de la philosophie transcendantale en ce qui concerne les passions fait que leur rôle est devenu délicat en philosophie politique, juridique, sociale, alors qu'il n'y a pas encore si longtemps il semblait plus facile à déterminer en ce domaine. En tout cas, ce Rousseau des temps modernes qui vient d'écrire un discours sur les sciences et les arts et sur l'origine de la rupture de l'équilibre des diverses figures de la culture nous doit encore une politique et un "Emile"; car il ne suffit pas de mettre en accusation le savoir galiléen comme fondement d'une monstrueuse "culture théorique " (p. 54), il faut encore définir un remède qui ne soit pas pire que le mal. Il parait que notre époque est celle du « triomphe de la politique » (p. 162). Est-ce tellement sûr?

Jean-Pierre ClERo. 